\title{
Molecular gas in the galaxy M 83
}

\section{The molecular gas distribution}

\author{
A. A. Lundgren ${ }^{1,2}$, T. Wiklind ${ }^{3,4}$, H. Olofsson ${ }^{1}$, and G. Rydbeck ${ }^{4}$ \\ 1 Stockholm Observatory, AlbaNova, 10691 Stockholm, Sweden \\ 2 European Southern Observatory, Casilla 19001, Santiago 19, Chile \\ 3 Space Telescope Science Institute, 3700 San Martin Drive Baltimore, MD 21218, USA \\ ${ }^{4}$ Onsala Space Observatory, 43992 Onsala, Sweden
}

Received 2 January 2003 / Accepted 22 September 2003

\begin{abstract}
We present ${ }^{12} \mathrm{CO} J=1-0$ and $J=2-1$ Swedish-ESO Submillimetre Telescope (SEST) observations of the barred spiral galaxy M 83 (NGC 5236). The size of the CO maps is $10^{\prime} \times 10^{\prime}$ and they cover the entire optical disk. The grid spacing is $11^{\prime \prime}$ for $\mathrm{CO}(J=1-0)$ and $11^{\prime \prime}$ or $7^{\prime \prime}$ for $\mathrm{CO}(J=2-1)$ depending on the position in the galaxy. In total we have obtained spectra in 1900 and 2574 positions in the $\mathrm{CO}(J=1-0)$ and $\mathrm{CO}(J=2-1)$ lines, respectively. The $\mathrm{CO}$ emission is strongly peaked toward the nucleus, which breaks up into two separate components in the $\operatorname{CO}(J=2-1)$ data due to the higher spatial resolution. Emission from the bar is strong, in particular on the leading edges of the bar. The molecular gas arms are clearly resolved and can be traced for more than $360^{\circ}$. Emission in the inter-arm regions is detected. The average $\mathrm{CO}(J=2-1) /(J=1-0)$ line ratio is 0.77 . The ratio is lower than this on the spiral arms and higher in the inter-arm regions. The arms show regularly spaced concentrations of molecular gas, Giant Molecular Associations (GMA's), whose masses are of the order $10^{7} M_{\odot}$. The total molecular gas mass is estimated to be $3.9 \times 10^{9} M_{\odot}$. This mass is comparable to the total HI mass, but $\mathrm{H}_{2}$ dominates in the optical disk. In the disk, $\mathrm{H}_{2}$ and $\mathrm{HI}$ show very similar distributions, including small scale clumping. We compare the molecular gas distribution with those of other star formation tracers, such as B and $\mathrm{H} \alpha$ images.
\end{abstract}

Key words. galaxies: individual: M 83; NGC 5236 - galaxies: spiral - galaxies: structure - galaxies: ISM radio lines: galaxies - galaxies: abundances

\section{Introduction}

In disk galaxies the mass of the molecular $\left(\mathrm{H}_{2}\right)$ gas is usually similar to, or a bit less than, that of the atomic (HI) gas (Young $\&$ Scoville 1991; Casoli et al. 1998). However, their spatial distributions are completely different, both in the radial and in the vertical directions. The molecular gas is concentrated to a relatively thin disk, which often has a radial extent similar to that of the optical disk and a vertical scale height which is smaller than that of the young, massive stars. The radius of the HI disk is usually much larger than that of the optical disk (up to 5 times the Holmberg radius), and the atomic gas is also less concentrated to the disk in the vertical direction (Knapp 1987).

If the mass surface density in a region exceeds a certain critical density, massive star formation is triggered (Kennicutt 1989). So, naturally, the study of interstellar gas, and especially the molecular gas phase, plays a key role in understanding the structure and evolution of galaxies. Unfortunately, direct studies (in emission) of the cold $(\sim 10 \mathrm{~K}) \mathrm{H}_{2}$ gas in molecular clouds are very difficult, since the energy of the first excited

Send offprint requests to: A. A. Lundgren,

e-mail: andreas@astro.su.se rotational level is of the order of $500 \mathrm{~K}$ (corresponding to a wavelength of $28 \mu \mathrm{m}$ ), and therefore much larger than the average kinetic temperatures in the clouds. Carbon monoxide (CO) is the second most common molecule with a relative abundance with respect to $\mathrm{H}_{2}$ of about $10^{-4}$. The heavy, asymmetrical $\mathrm{CO}$ molecule has rotational levels which are easily excited by collisions with $\mathrm{H}_{2}$, and it radiates at mm wavelengths where observations can be carried out using radio telescopes. However, the conversion factor which is used to derive the $\mathrm{H}_{2}$ column density from the velocity-integrated $\mathrm{CO}$ intensity, $X_{\mathrm{CO}}$, is not well-known, and this is a source of major uncertainty (Young \& Scoville 1991; Combes 1991).

$\mathrm{CO}$ emission at mm wavelengths has been detected in many galaxies ranging in distance from the nearby irregular galaxies The Large and The Small Magellanic Clouds to quasars at a redshift of more than $z \sim 5$, and in essentially all galaxy types. A large survey of 300 galaxies (of which 236 were detected) of different types was done by Young et al. (1995) using the FCRAO $14 \mathrm{~m}$ telescope. The analysis of the data is still ongoing, but some interesting results have been published, e.g., the conversion factor $\left(X_{\mathrm{CO}}\right)$ appears relatively constant, at least within each galaxy type (Young \& Rownd 2001), and the global 
star formation efficiency decreases with increasing galaxy size (Young 1999). In this survey a number of galaxies were also mapped and radial distributions of the molecular gas can be examined. Recently, in a more detailed study, 28 nearby galaxies were mapped with the $45 \mathrm{~m}$ Nobeyama telescope (Kuno et al. 2000; Nishiyama et al. 2001). Among other things, this showed that barred and non-barred galaxies differ in both the distribution and the kinematics of the molecular gas. Another large survey is the BIMA SONG project (Regan et al. 2001; Helfer et al. 2003), in which 44 nearby $\left(v_{\odot}<2000 \mathrm{~km} \mathrm{~s}^{-1}\right)$ galaxies have been observed with the BIMA array and the NRAO $12 \mathrm{~m}$ telescope. Early results show that many galaxies have multiple peaks at the nucleus, that the $\mathrm{CO}$ distribution usually cannot be fitted with a single exponential disk model, and that the ratio of the scale length in the $\mathrm{CO}$ disk and the scale length in the stellar disk is on average 1 (but the scatter around this average is large).

Detailed morphological studies of selected galaxies are more rare, partly because there are so few nearby galaxies for which the limited angular resolution of radio telescopes gives an adequate spatial resolution. Nakai et al. (1994) outlined a number of important observational conditions that should be fulfilled in order to be able to extract useful information on the properties of the molecular gas in different regions of a galaxy: (1) a spatial resolution better than one kpc, which is the typical width of molecular spiral arms and the spacing between them, (2) high sensitivity in order to detect the weak inter-arm emission, (3) mapping of a large field in order to get statistically meaningful results, and (4) filled aperture telescope data, since it is important to detect the diffuse emission in the inter-arm regions. To this list we add a fifth condition, (5) the observation should be carried out in (at least) two lines per molecular species (and preferably also partially observed in a few isotopomers) in order to gain insight into the density, temperature, and chemistry of the molecular gas.

Single-dish measurements are therefore essential to our determination of the molecular gas distribution, its relation to star formation activity, its location with respect to dynamical resonances, and the total reservoir of gas capable of forming future generations of stars. The most detailed single-dish maps of molecular gas in spiral galaxies are the mappings of M 51, using the IRAM 30 m telescope (Garcia-Burillo et al. 1993) and the Nobeyama $45 \mathrm{~m}$ telescope (Nakai et al. 1994), and two projects currently underway to map our neighbor M31 (Loinard et al. 1999; Nieten et al. 2000). In M 51 the molecular spiral arms were resolved, and streaming motions were detected. Also, the $\mathrm{CO}$ emission was found to peak on the inside of the spiral arms, giving support to a scenario where molecular gas agglomerates and forms new stars, which in their turn produce $\mathrm{H} \alpha$ emission and other star formation tracers, close to the spiral arms. Support was also found for the proposal that gravitational instability in the disk is the main formation mechanism of GMAs (Galactic Molecular Associations of mass $\sim 10^{7} M_{\odot}$ ). Other large spiral galaxies that have been completely surveyed in $\mathrm{CO}$ with a single-dish telescope are IC 342 (Crosthwaite et al. 2001), M 101 (Kenney et al. 1991), NGC 253 (Houghton et al. 1997), and NGC 6946 (Tacconi \& Young 1989). Some large scale CO mapping projects, such as the OVRO maps
Table 1. General parameters of M 83.

\begin{tabular}{ll}
\hline \hline Morphological type $^{a}$ & $\mathrm{SAB}(\mathrm{s}) \mathrm{c}$ \\
IR center: $^{b}$ & \\
$\mathrm{RA}(\mathrm{J} 2000)$ & $13^{\mathrm{h}} 37^{\mathrm{m}} 00^{\circ} .8$ \\
Dec (J2000) & $-29^{\circ} 51^{\prime} 56^{\prime \prime}$ \\
LSR systemic velocity (opt) $^{c}$ & $506 \mathrm{~km} \mathrm{~s}^{-1}$ \\
Distance $^{d}$ & $4.5 \mathrm{Mpc}$ \\
Position angle $^{c}$ & $45^{\circ}$ \\
Inclination $^{c}$ & $24^{\circ}$ \\
${\text { Holmberg diameter }\left(D_{0}\right)^{f}}^{\circ}$ & 14.6 \\
$M_{\mathrm{HI}}^{e}$ & $7.7 \times 10^{9} M_{\odot}$ \\
$M_{\mathrm{H}_{2}}^{g}$ & $3.9 \times 10^{9} M_{\odot}$ \\
\hline
\end{tabular}

${ }^{a}$ de Vaucouleurs et al. (1976); ${ }^{b}$ Sofue \& Wakamatsu (1994); ${ }^{c}$ Comte (1981); ${ }^{d}$ Thim et al. (2003); ${ }^{e}$ Huchtmeier \& Bohnenstengel (1981); ${ }^{f}$ Talbot et al. (1979); ${ }^{g}$ this paper.

of M 51 (Aalto et al. 1999) and M 83 (Rand et al. 1999), have been done using mm-wave interferometers. Although this gives a superior angular resolution and important unique information, a significant part of the emission is resolved out (especially the diffuse component in the inter-arm regions).

We have mapped the $\operatorname{CO}(J=1-0)$ and $\operatorname{CO}(J=2-1)$ emission in the barred, grand-design, spiral galaxy M 83 located at a distance of $4.5 \mathrm{Mpc}$. The observations were done with the $15 \mathrm{~m}$ Swedish-ESO Submillimetre Telescope (SEST) providing beam widths of $45^{\prime \prime}(1-0)$ and $23^{\prime \prime}(2-1)$, which corresponds to $980 \mathrm{pc}$ and $500 \mathrm{pc}$, respectively, at the adopted distance. M 83 is among the brightest galaxies in terms of $\mathrm{CO}$ emission, and it is also one of the most nearby barred spirals. Its low inclination $\left(\approx 24^{\circ}\right)$ and proximity make it a perfect candidate to investigate the correlation between various tracers of stellar activity.

In this paper we present the $\mathrm{CO}(J=1-0)$ and $\mathrm{CO}(J=2-1)$ observational data. We show the distribution of the molecular gas, as inferred from the CO data, and compare it to the distributions of optical light, obtained in different filters, and the HI gas. We examine the $\mathrm{CO}(J=2-1) /(J=1-0)$ line ratio and its variation over the disk. In two forth-coming papers we will present and discuss the kinematics of the molecular gas, and discuss the relation between the molecular gas and star formation.

\section{The galaxy M 83}

M 83 is a relatively nearby, barred, grand-design, spiral galaxy (see Table 1). It is viewed almost face-on and the bar is aligned with the major axis. Color images show clumpy, well-defined spiral arms, evidently rich in young blue stars. It is also fairly symmetrical and has no nearby massive optical companions and no immediate evidence of interaction or outflows.

Due to its favorable properties it is one of the most wellobserved galaxies: HI (Rogstad et al. 1974; Huchtmeier \& Bohnenstengel 1981; Tilanus \& Allen 1993), radio continuum 
Table 2. Previous CO observations of M 83 divided into two groups: single-dish and interferometer observations. If no location is given, the map is centered on the nucleus and oriented along the major axis.

\begin{tabular}{|c|c|c|c|}
\hline Reference & Transition & Beam and telescope & Map description \\
\hline Combes et al. (1978) & $\mathrm{CO}(1-0)$ & $64^{\prime \prime}$, NRAO $11 \mathrm{~m}$ & 10 positions, $6^{\prime} \times 3^{\prime}$ \\
\hline Lord (1987) & $\mathrm{CO}(1-0)$ & $45^{\prime \prime}$, FCRAO $14 \mathrm{~m}$ & 21 positions, $5^{\prime} \times 5^{\prime}$ \\
\hline \multirow[t]{2}{*}{ Wiklind et al. (1990) } & $\mathrm{CO}(1-0)$ & $45^{\prime \prime}$, SEST $15 \mathrm{~m}$ & 196 positions, SE quadrant, $3^{\prime} \times 2^{\prime}$ \\
\hline & $\mathrm{CO}(2-1)$ & $23^{\prime \prime}$, SEST $15 \mathrm{~m}$ & 143 positions, SE quadrant, $1.5 \times 1{ }^{\prime} .5$ \\
\hline Handa et al. (1990) & $\mathrm{CO}(1-0)$ & $16^{\prime \prime}$, NRO $45 \mathrm{~m}$ & 85 positions, $3{ }^{\prime} .5 \times 1^{\prime}$ \\
\hline \multirow[t]{2}{*}{ Wall (1991) } & $\mathrm{CO}(2-1)$ & $22^{\prime \prime}$, JCMT $15 \mathrm{~m}$ & 43 positions \\
\hline & $\mathrm{CO}(3-2)$ & $22^{\prime \prime}$, CSO $10 \mathrm{~m}$ & 8 positions \\
\hline \multirow[t]{2}{*}{ Petitpas \& Wilson (1998) } & $\mathrm{CO}(3-2)$ & $14^{\prime \prime}$, JCMT $15 \mathrm{~m}$ & 81 positions, $0{ }^{\prime} 7 \times 0.7$ \\
\hline & $\mathrm{CO}(4-3)$ & $12^{\prime \prime}$, JCMT $15 \mathrm{~m}$ & 46 positions, $0{ }^{\prime} .7 \times 0.5$ \\
\hline \multirow[t]{3}{*}{ Israel \& Baas (2001) } & $\mathrm{CO}(2-1)$ & $21^{\prime \prime}$, JCMT $15 \mathrm{~m}$ & 49 positions, $1.2 \times 2{ }^{\prime} .0$ \\
\hline & $\mathrm{CO}(3-2)$ & $14^{\prime \prime}$, JCMT $15 \mathrm{~m}$ & 55 positions, $1.2 \times 1.7$ \\
\hline & $\mathrm{CO}(4-3)$ & $11^{\prime \prime}$, JCMT $15 \mathrm{~m}$ & 20 positions, $0.5 \times 0.5$ \\
\hline \multirow[t]{2}{*}{ Crosthwaite et al. (2002) } & $\mathrm{CO}(1-0)$ & $55^{\prime \prime}$, NRAO $12 \mathrm{~m}$ & On-the-fly, $10^{\prime} \times 10^{\prime}$ \\
\hline & $\mathrm{CO}(2-1)$ & $28^{\prime \prime}$, NRAO $12 \mathrm{~m}$ & On-the-fly, $8^{\prime} \times 8^{\prime}$ \\
\hline \multirow[t]{2}{*}{ Thuma et al. (in prep.) } & $\mathrm{CO}(3-2)$ & $23^{\prime \prime}$, SMT $10 \mathrm{~m}$ & 374 positions, $5.5 \times 2.5$ \\
\hline & $\mathrm{CO}(4-3)$ & 17", SMT $10 \mathrm{~m}$ & 21 positions \\
\hline \multirow[t]{2}{*}{ This paper } & $\mathrm{CO}(1-0)$ & $45^{\prime \prime}$, SEST $15 \mathrm{~m}$ & 1900 positions, $10^{\prime} \times 10^{\prime}$ \\
\hline & $\mathrm{CO}(2-1)$ & $23^{\prime \prime}$, SEST $15 \mathrm{~m}$ & 2574 positions, $10^{\prime} \times 10^{\prime}$ \\
\hline Lord \& Kenney (1991) & $\mathrm{CO}(1-0)$ & $12^{\prime \prime} 4 \times 5^{\prime \prime} .4$, OVRO & Inner eastern arm, $1.5 \times 1.5$ \\
\hline Kenney \& Lord (1991) & $\mathrm{CO}(1-0)$ & $8.9 \times 55^{\prime \prime} 8$, OVRO & Western bar end, $1.5 \times 1{ }^{\prime} .5$ \\
\hline Handa et al. (1994) & $\mathrm{CO}(1-0)$ & $12^{\prime \prime} \times 6^{\prime \prime}, \mathrm{NMA}$ & Nucleus, $1^{\prime}: 1 \times 1{ }^{\prime} 1$ \\
\hline Rand et al. (1999) & $\mathrm{CO}(1-0)$ & $6^{\prime \prime} 5 \times 33^{\prime \prime} 5$, OVRO & Inner eastern arm, $2^{\prime} .9 \times 1^{\prime} .1$ \\
\hline
\end{tabular}

(Ondrechen 1985; Neininger et al. 1993), CO (see Table 2), IR continuum (Adamson et al. 1987; Fitt et al. 1992; Elmegreen et al. 1998), optical continuum (Jensen et al. 1981), Balmer lines (Comte 1981; de Vaucouleurs et al. 1983; Tilanus \& Allen 1993), and in X-ray emission (Immler et al. 1999; Soria \& Wu 2002).

The distance to M 83 is a long-standing issue. Sandage \& Tammann (1974) used a relation between the luminosity class and the angular size of the three largest HII regions to deduce a distance of $8.9 \mathrm{Mpc}$, while de Vaucouleurs (1979) used a number of methods to derive a distance of $3.7 \mathrm{Mpc}$. Recently, Cepheids have been observed in M 83 by Thim et al. (2003) using the VLT. Twelve Cepheid candidates were observed and the distance was estimated to be $4.5 \pm 0.3 \mathrm{Mpc}$. This distance is also supported by studies that seem to indicate that M 83 interacted with NGC 5253 some 1-2×109 years ago (Kobulnicky \& Skillman 1995; Calzetti et al. 1999). This small, metal-poor dwarf galaxy about $2^{\circ}$ SE of M 83 has roughly the same systemic velocity, and shows a highly disturbed HI velocity field and intense star formation. There has been a number of distance measurements to NGC 5253 (Saha et al. 1995; Parodi et al. 2000; Gibson et al. 2000), and they all favor a distance of about $4 \mathrm{Mpc}$.

\section{Observations and data reduction}

\subsection{Observations}

The $\operatorname{CO}(J=1-0$ and $J=2-1)$ observations of M 83 were done using the $15 \mathrm{~m} \mathrm{SEST}^{1}$ during two epochs, 1989-1994 and 1997-2001 (a description of the telescope is given by Booth et al. 1989). Between 1988 and 1994 we observed $\mathrm{CO}(J=1-0)$ in the inner disk of M 83. A segment of these observations were presented in Wiklind et al. (1990). In 1998 and 1999 we completed this map by observing the outer disk. During the latter observations it was possible to do simultaneous observations in two frequency bands. We used this option to observe the $\operatorname{CO}(J=2-1)$ line in parallel with the $\mathrm{CO}(J=1-0)$ line. The map spacing $\left(11^{\prime \prime}\right)$ and integration times were set by the requirements on the $\operatorname{CO}(J=1-0)$ data. In 2000 and 2001 our primary task was to fill in the central area of the $\operatorname{CO}(J=2-1)$ map. For these observations we chose a grid spacing of $7 "$. We used the other receiver to observe the ${ }^{13} \mathrm{CO}(J=1-0)$ line, but these data will be presented elsewhere.

\footnotetext{
${ }^{1}$ The Swedish-ESO Submillimetre Telescope is operated jointly by ESO and the Swedish National Facility for Radio Astronomy, Chalmers University of Technology.
} 
Table 3. SEST observation parameters for M 83.

\begin{tabular}{lll}
\hline \hline & $\mathrm{CO}(J=1-0)$ & $\mathrm{CO}(J=2-1)$ \\
\hline Center of the CO map & $\mathrm{RA}=13^{\mathrm{h}} 36^{\mathrm{m}} 59^{\mathrm{s}} .4(\mathrm{~J} 2000)$ \\
& $\mathrm{Dec}=-29^{\circ} 52^{\prime} 05^{\prime \prime}(\mathrm{J} 2000)$ \\
Rest frequency & $115.271204 \mathrm{GHz}$ & $230.53799 \mathrm{GHz}$ \\
Beam size & $45^{\prime \prime}$ & $23^{\prime \prime}$ \\
Center velocity $(\mathrm{LSR})$ & $520 \mathrm{~km} \mathrm{~s}^{-1}$ & $520 \mathrm{~km} \mathrm{~s}^{-1}$ \\
Average rms $\left(T_{\mathrm{mb}}\right.$ scale $)$ & $74 \mathrm{mK}$ & $90 \mathrm{mK}$ \\
Channel separation & $\leq 0.69 \mathrm{MHz}$ & $0.69 \mathrm{MHz}$ \\
Number of channels & $\geq 700$ & 1000 \\
Main beam efficiency $\left(\eta_{\mathrm{mb}}\right)$ & 0.7 & 0.5 \\
Aperture efficiency $\left(\eta_{\mathrm{A}}\right)$ & $0.58(27 \mathrm{Jy} / \mathrm{K})$ & $0.38(41 \mathrm{Jy} / \mathrm{K})$ \\
\hline
\end{tabular}

The receivers were centered on the $\operatorname{CO}(J=1-0)$ and $\mathrm{CO}(J=2-1)$ lines $(115.271$ and $230.538 \mathrm{GHz}$, respectively), where the full half-power beam widths (HPBWs) are $45^{\prime \prime}$ and $23^{\prime \prime}$, respectively. Observations performed during 1988-1994 were made with a cooled Schottky-diode mixer receiver, while the subsequent observations were done with SIS receivers. All receivers operated in the single-sideband mode, and the average system temperatures, corrected for the atmospheric and antenna ohmic losses, were $660 \mathrm{~K}$ and $390 \mathrm{~K}$ for the $\operatorname{CO}(J=1-0)$ observations in the first and second epoch, respectively, and $340 \mathrm{~K}$ for the $\mathrm{CO}(J=2-1)$ observations. The backends were acousto-optical spectrometers, with channel separations of $0.69 \mathrm{MHz}\left(1.8\right.$ and $0.9 \mathrm{~km} \mathrm{~s}^{-1}$ at the two rest frequencies) and total bandwidths varying between 0.5 and $1 \mathrm{GHz}$.

We used the dual beam switching mode with a throw of $\approx 12^{\prime}$ in azimuth. In dual beam switching the source is placed first in the signal beam and then in the reference beam. The two spectra produced are then subtracted to generate a spectrum with a minimum of baseline variation. Great care was taken to ensure the quality of the data. No observations were done below the elevation of $30^{\circ}$ or above $84^{\circ}$ (M 83 passes within $1^{\circ}$ from zenith at SEST), and pointing was regularly checked and updated using the $\operatorname{SiO}(v=1, J=2-1)$ maser line of the nearby AGB-star W Hya $\left(\approx 3^{\circ}\right.$ from M 83). Total pointing errors were typically less than $3^{\prime \prime}$, and data with pointing offsets suspected to be larger than $4^{\prime \prime}$ were disregarded in the subsequent data reduction (when possible, map points with such data were reobserved). The intensity scale was calibrated using the conventional chopper wheel method, and the internal calibration errors in the corrected antenna temperatures, $T_{\mathrm{A}}^{*}$, is within $\pm 10 \%$ according to the SEST manual. In this paper we use the main beam brightness temperature scale $T_{\mathrm{mb}}$, which is defined by $T_{\mathrm{mb}} \equiv T_{\mathrm{A}}^{*} / \eta_{\mathrm{mb}}$. According to the SEST manual the main beam efficiencies $\left(\eta_{\mathrm{mb}}(115 \mathrm{GHz})=0.7\right.$, $\left.\eta_{\mathrm{mb}}(230 \mathrm{GHz})=0.5\right)$ have been constant throughout the period of our observations.

The observations were centered on the coordinates $13^{\mathrm{h}} 36^{\mathrm{m}} 59^{\mathrm{s}} .4,-29^{\circ} 52^{\prime} 05^{\prime \prime}(\mathrm{J} 2000)$, which is the optical center given by de Vaucouleurs et al. (1976). For $\operatorname{CO}(J=1-0)$ we have 3634 spectra in 1900 positions with $11^{\prime \prime}$ spacing, and the coverage is complete out to a radius of $4^{\prime} 20^{\prime \prime}$. The grid extends parallel to the equatorial coordinate system, and the dense spacing (1/4 of the HPBW) was chosen in order to facilitate the use of deconvolution techniques. The integration time per position was chosen to render an rms around $70 \mathrm{mK}$ ( $T_{\mathrm{mb}}$-scale) at the original velocity resolution $\left(1.8 \mathrm{~km} \mathrm{~s}^{-1}\right)$. During normal weather conditions we had a typical on-source integration time of $60 \mathrm{~s}$. Among the final 1900 spectra we have $\geq 3 \sigma$ detections in 1201 positions, i.e. a detection rate of $63 \%$. The average rms noise in our data set is $74 \mathrm{mK}$, which should be compared to the peak temperature which usually lies around $0.3 \mathrm{~K}$ ( $T_{\mathrm{mb}}$-scale) in the spiral arms in the outskirts of the map.

In $\mathrm{CO}(J=2-1)$ we have two maps with different spacings covering two, partly overlapping, regions. The inner $5^{\prime} \times 3^{\prime}$ is covered with a $7^{\prime \prime}$ grid, while the rest of the optical disk (out to a radius of 4.5) is covered with an $11^{\prime \prime}$ grid. In total, we have spectra in 2574 positions, out of which we have $\geq 3 \sigma$ detections in 1898 positions ( $73 \%$ detection rate). The spectra have an average rms of about $90 \mathrm{mK}$ at the original velocity resolution $\left(0.9 \mathrm{~km} \mathrm{~s}^{-1}\right)$. The observational parameters are summarized in Table 3 .

\subsection{Data reduction}

The data reduction was carried out with $\mathrm{CLASS}^{2}, \mathrm{DRP}^{3}$, and $\mathrm{XS}^{4}$. During the $\mathrm{CO}(J=1-0)$ observations, the number of channels and the frequency resolution varied between the spectrometers. We therefore resampled all data to $1.8 \mathrm{~km} \mathrm{~s}^{-1}(0.69 \mathrm{MHz})$ and by selecting the central 700 channels this gave us a (fully sufficient) total bandwidth of $1260 \mathrm{~km} \mathrm{~s}^{-1}$.

After flagging bad channels, and replacing them with a value obtained from interpolation between adjacent channels, all spectra at the same position were averaged using a weight

\footnotetext{
${ }^{2}$ Continuum and Line Analysis Single-dish Software from the Observatoire de Grenoble and Institut de Radio-Astronomie Millimétrique.

${ }^{3}$ Data Reduction Package developed at Onsala Space Observatory.

${ }^{4}$ xs: Spectral Line Reduction Package for Astronomy developed at Onsala Space Observatory.
} 
determined by the noise level. The baselines were stable, and only linear polynomial fits were subtracted. From the spectra we created a FITS data cube for each transition, where the first two axes represent spatial coordinates, and the third axis represents frequency (or velocity).

We adopted a relatively dense grid spacing, compared to the beamwidth, in order to be able to increase the spatial resolution of the data using a MEM-deconvolution routine (maximum entropy method); the Statistical Image Analysis (SIA) routine (Rydbeck 2000). This procedure is particularly effective on a data set as densely spaced as ours (spacing in the range $1 / 4-1 / 2$ of the HPBW), due to the high redundancy. The routine starts by deconvolving a velocity-integrated intensity map, where the velocity range is chosen to be large enough to encompass all the emission. Once this is done, the procedure splits the initial velocity range into three equally wide regions, calculates their velocity-integrated intensities, and then deconvolves these three maps, using the deconvolved map from the previous step as the input guess. The deconvolution continues in this hierarchical manner, successively narrowing the velocity interval, until a velocity range of $5 \mathrm{~km} \mathrm{~s}^{-1}$ is reached. To deconvolve beyond this point is not meaningful since the $\mathrm{S} / \mathrm{N}$-ratios in the (narrow velocity range) maps drop below a critical value. For each of the two CO transitions we created $81\left(3^{4}\right)$ maps per transition (each covering about $5 \mathrm{~km} \mathrm{~s}^{-1}$ ) which we assembled into a data cube per transition. By convolving the MEM-deconvolved data cube by a Gaussian with a FWHM equal to the observational HPBW and comparing with the raw spectra, we verified that the MEM-results are reliable, and that the raw data set is homogeneous, i.e., free from variations in gain and pointing errors. The angular resolutions of the MEM-cubes are estimated to be $\approx 22^{\prime \prime}$ and $\approx 14^{\prime \prime}$ for the $\mathrm{CO}(J=1-0)$ and $\operatorname{CO}(J=2-1)$ data, respectively, and the velocity resolution is $5 \mathrm{~km} \mathrm{~s}^{-1}$.

We have also constructed convolved data sets by convolving the raw data cubes with a HPBW beam size of $20^{\prime \prime}$ and $15^{\prime \prime}$ for the $\operatorname{CO}(J=1-0)$ and $\operatorname{CO}(J=2-1)$ data, respectively (the spacing in these cubes are $\left.11^{\prime \prime}\right)$. Even if this degrades the resolution by $\approx 10-20 \%$, the average rms noise in these cubes decreases from $74 \mathrm{mK}$ to $25 \mathrm{mK}$ and from $90 \mathrm{mK}$ to $31 \mathrm{mK}$, respectively. The dramatically decreased noise level is due to the fact that the spacing is small with respect to the HPBW of the convolution kernels. Since the peak intensity in the spectra are relatively unaffected by this process, the average $\mathrm{S} / \mathrm{N}$-ratio in the spectra increases by a factor of $\approx 3$. As a result of this, the detection rate increases to $92 \%$ and $89 \%$ for the $\operatorname{CO}(J=1-0)$ and $\operatorname{CO}(J=2-1)$ data, respectively (cf. Sect. 3.1). These cubes were used to cross-check the results we got from the MEM-deconvolved data. Some results were also derived directly from the convolved cubes, especially in cases where the velocity resolution was more important than the spatial resolution. Furthermore, we convolved the raw $\mathrm{CO}(J=2-1)$ cube with a beam size of $44^{\prime \prime}$ and regridded it to $11^{\prime \prime}$ spacing in order to compare with the convolved $\mathrm{CO}(J=1-0)$ cube at the same resolution $\left(\approx 49^{\prime \prime}\right)$.

In order to avoid problems with baseline irregularities when calculating the zero moment (over velocity) of the intensities we used a sliding window technique where at each position integration was performed inside a velocity window whose center and width depend on the position within the galaxy. We checked carefully that the window always covered all the emission.

\subsection{Correction for the error beam}

A closer inspection of the $\mathrm{CO}(J=2-1)$ spectra reveals, apart from the expected main component seen in the $\operatorname{CO}(J=1-0)$ spectra, a broad (velocity width $400 \mathrm{~km} \mathrm{~s}^{-1}$ ) and faint (peak intensity around $0.03 \mathrm{~K}$ ) component. Despite the low peak intensity of this feature, its integrated intensity is quite appreciable due to its large width. Typically, it makes up to $20 \%-35 \%$ of the total integrated intensity. This feature was discovered in the MEM-deconvolved data, which are noise-free, but it can be seen also in spectra produced by convolving the raw $\mathrm{CO}(J=2-1)$ data cube with a $45^{\prime \prime}$ beam. The appearance of this broad component changed with position in the disk, and we found that its shape could, in any given position, be reproduced very well by convolving the data set with a relatively large Gaussian beam. This indicates that the SEST picks up emission from a much larger area than the HPBW of $23^{\prime \prime}$, i.e., at $230 \mathrm{GHz}$ the SEST suffers from a non-negligible error beam.

We have corrected the $\mathrm{CO}(J=2-1)$ data sets for this error using a method described in Appendix A. We have found no trace of an error beam contribution in the $\mathrm{CO}(J=1-0)$ data (not unexpectedly, considering the higher main beam efficiency), and consequently we have not applied any correction to these data.

\section{The data}

\subsection{Spectra}

In general, the spectra have a smooth and symmetric shape, but in some areas they show deviations. The spectra close to the nucleus are asymmetric, show extended wings, and a few of them have multiple peaks. The main reason for this is a large velocity gradient over spatial scales small compared to the telescope beam. Further out in the disk there are a few (interarm) regions which show multiple-peak spectra, such as the "Gould-belt structure" located about 3' SE of the galaxy center (Comerón 2001). However, in the vast majority of the observed positions the spectral feature can be well described by a single, Gaussian profile.

\subsubsection{The $\operatorname{CO}(J=1-0)$ spectra}

In the arms we find that the peak temperatures are typically $0.2-0.4 \mathrm{~K}$, and in the inter-arm regions they are about $0.1 \mathrm{~K}$. The spectra are widest in the nuclear region with (Gaussian) widths of $80-100 \mathrm{~km} \mathrm{~s}^{-1}$. Further out the linewidths are of the order of $7-14 \mathrm{~km} \mathrm{~s}^{-1}$ in the arms, with no systematic difference between arm and inter-arm regions.

The global $\operatorname{CO}(J=1-0)$ spectrum (left panel in Fig. 1) has a velocity-integrated intensity of $8.18 \pm 0.04 \mathrm{~K} \mathrm{~km} \mathrm{~s}^{-1}$ (excluding the calibration error) and a noise level of $1.8 \mathrm{mK}$ (all errors in this paper are $3 \sigma$ unless otherwise noted). The receding part 

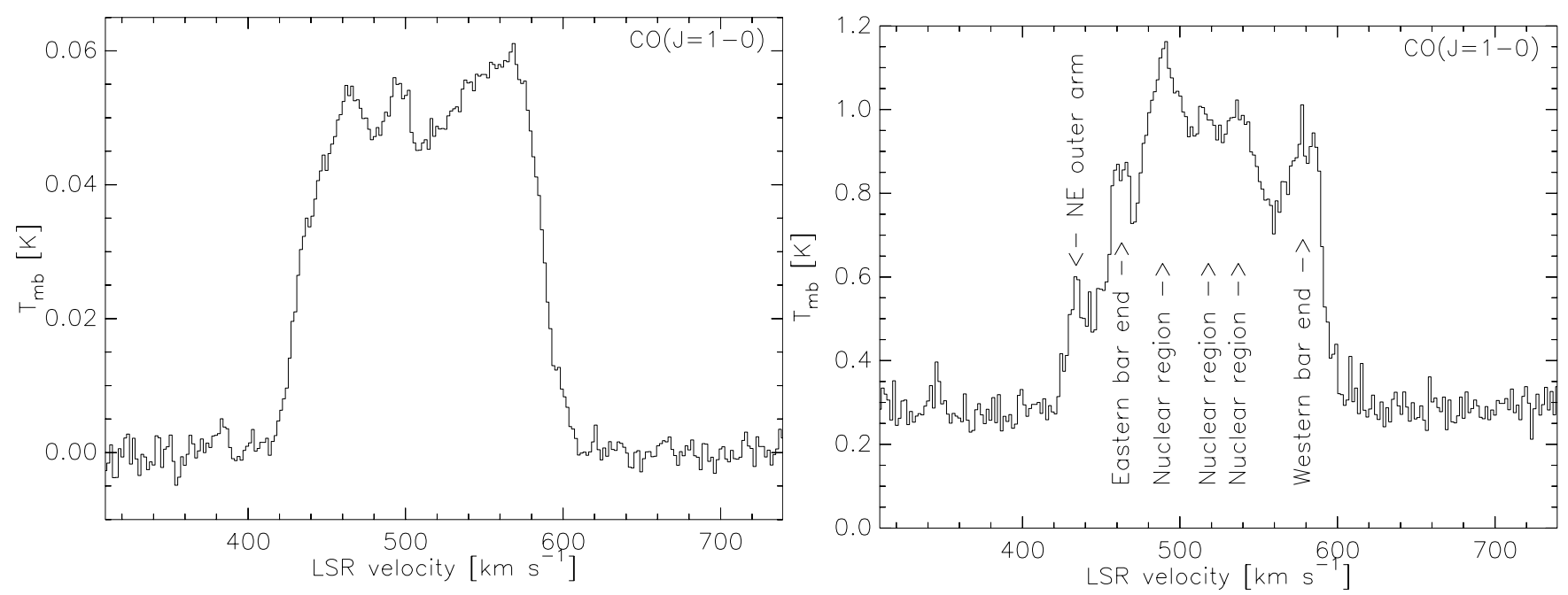

Fig. 1. Left: Global $\mathrm{CO}(J=1-0)$ spectrum. Right: Peak intensity $\mathrm{CO}(J=1-0)$ spectrum.
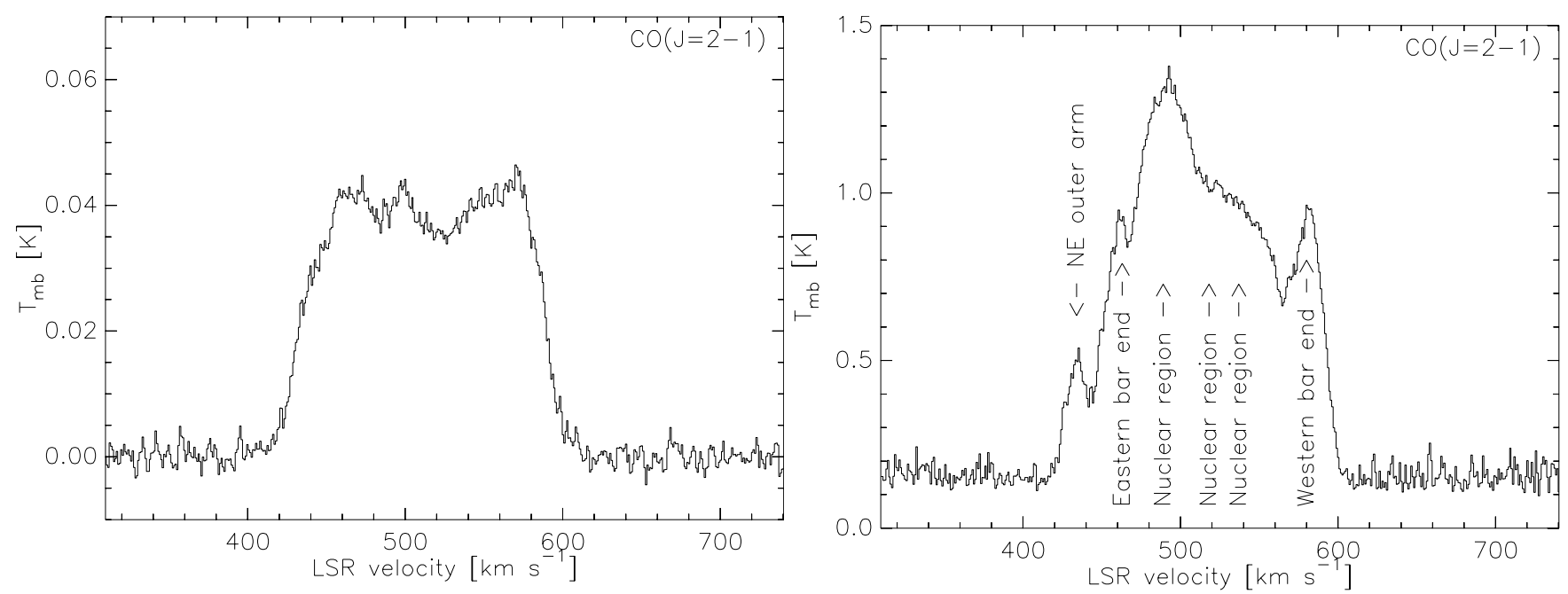

Fig. 2. Left: Global $\mathrm{CO}(J=2-1)$ spectrum. Right: Peak intensity $\mathrm{CO}(J=2-1)$ spectrum. Before producing these spectra the $\mathrm{CO}(J=2-1)$ data cube was regridded to $11^{\prime \prime}$ spacing.

of the galaxy (SW) has more $\mathrm{CO}(J=1-0)$ emission than the approaching part (NE): the velocity interval $510-610 \mathrm{~km} \mathrm{~s}^{-1}$ is $18 \%$ more luminous than the $410-510 \mathrm{~km} \mathrm{~s}^{-1}$ interval. An excess of higher-velocity emission has also been seen in the atomic gas. Huchtmeier \& Bohnenstengel (1981) found that the higher-velocity part of the $\mathrm{HI}$ emission is $16 \%$ more luminous than the lower-velocity part.

The peak intensity spectrum (Fig. 1, right panel) has been obtained by selecting, for every channel, the highest intensity in the map. Therefore, the noise level in this spectrum reflects the noise in the overall worst case. From studies of the channel maps we conclude that the peaks in this spectrum arise from the nuclear region $\left(487,514\right.$, and $\left.534 \mathrm{~km} \mathrm{~s}^{-1}\right)$, the western bar end $\left(580 \mathrm{~km} \mathrm{~s}^{-1}\right)$, the eastern bar end $\left(460 \mathrm{~km} \mathrm{~s}^{-1}\right)$, and the NE outer spiral arm $\left(437 \mathrm{~km} \mathrm{~s}^{-1}\right)$.

By adding the $\operatorname{CO}(J=1-0)$ spectra (from the raw data cube) in concentric, $20^{\prime \prime}$ wide annuli (compensated for inclination and position angle and centered on the IR nucleus, see Table 1), we produced the spectra in Fig. 3. They show how the average $\mathrm{CO}(J=1-0)$ velocity-integrated intensity changes with galactocentric radius (which is indicated, in arc-seconds, in the upper right corner of each panel). The coverage in the map is complete to a radius of $229^{\prime \prime}$.

\subsubsection{The $\operatorname{CO}(J=2-1)$ spectra}

The global and peak intensity $\mathrm{CO}(J=2-1)$ spectra show characteristics very similar to those of the $\mathrm{CO}(J=1-0)$ data, Fig. 2 (since the $\mathrm{CO}(J=2-1)$ spectra were obtained with two different spacings, we regridded these data to a common $11^{\prime \prime}$ spacing before producing the spectra in Fig. 2). The main difference is that the peak intensity spectrum is brighter than the corresponding $\mathrm{CO}(J=1-0)$ spectrum. This is most likely an effect of the emitting objects being smaller than the $\mathrm{CO}(J=1-0)$ beam.

In Table 4 we summarize the characteristics of the global spectra. The global line intensity ratio $R_{21} \equiv$ $\int T_{\mathrm{mb}}(2-1) \mathrm{d} v / \int T_{\mathrm{mb}}(1-0) \mathrm{d} v$ is $0.77 \pm 0.01$, based on the errors from Table 4. Note that, including errors in the calibration and the main beam and error beam efficiencies, the result is $0.77 \pm 0.16$. However, it should be noted that the relative 

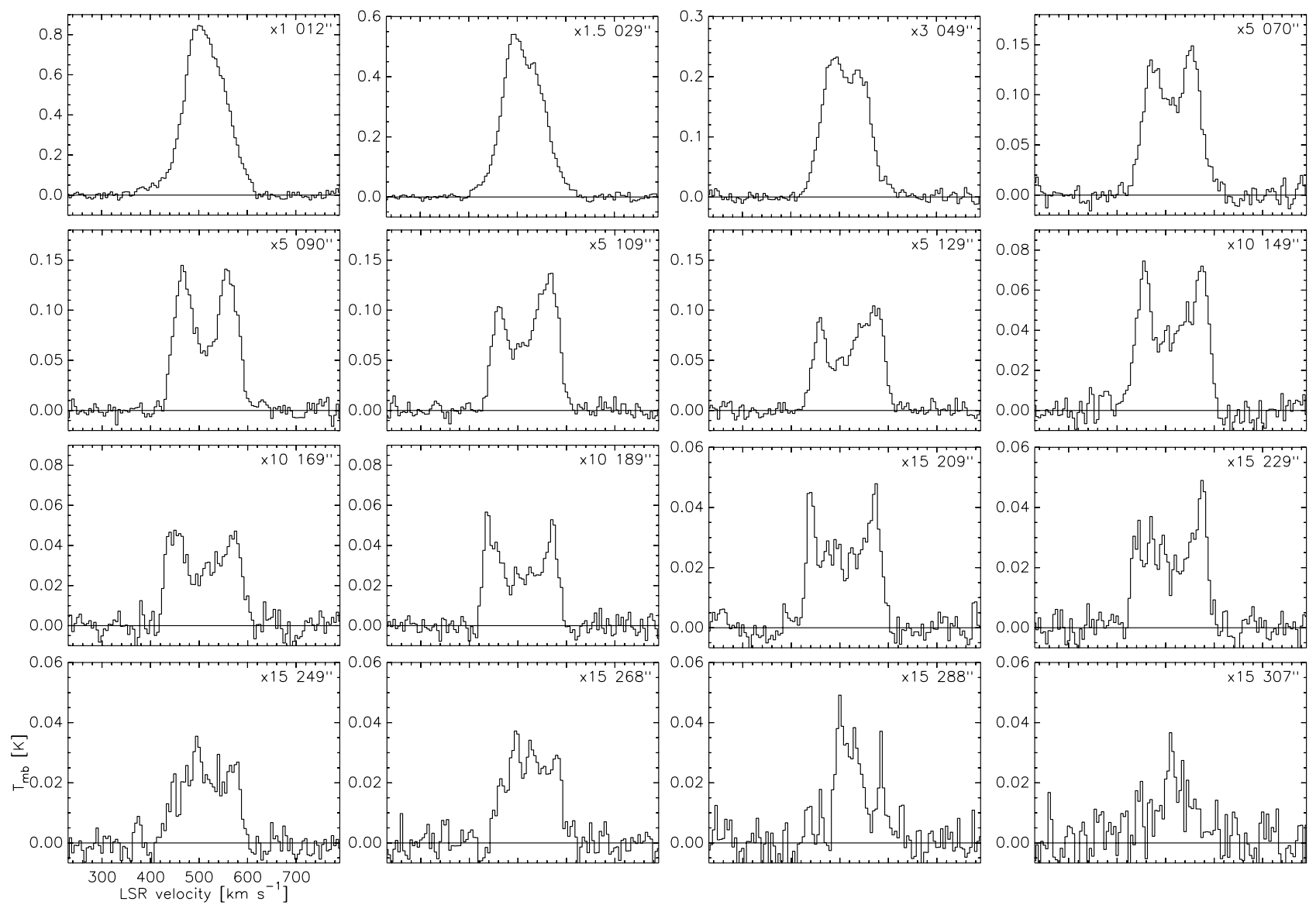

Fig. 3. $\mathrm{CO}(J=1-0)$ spectra produced by averaging all spectra within successive, concentric (and inclination-corrected) annuli. The average radius (in arc-seconds) of each annuli is shown in the upper right corner. The center spectrum is shown in the upper left corner, and the outermost spectrum in the lower right corner. Note that the intensity scale varies from panel to panel (the scaling factor is shown in the upper right corner of each panel). The velocity resolution has in this image been reduced to $5 \mathrm{~km} \mathrm{~s}^{-1}$.

Table 4. Peak intensities, velocity-integrated intensities (with errors), intensity-weighted velocities, and equivalent widths of the global $\mathrm{CO}(J=1-0)$ and $\operatorname{CO}(J=2-1)$ spectra in Figs. 1 and 2. The errors are estimated from the noise levels. In the column for integrated intensities we also show the absolute errors.

\begin{tabular}{ccccc}
\hline \hline & $\begin{array}{c}\text { Peak } \\
{[\mathrm{K}]}\end{array}$ & $\begin{array}{c}\text { Integral } \\
{\left[\mathrm{K} \mathrm{km} \mathrm{s}^{-1}\right]}\end{array}$ & $\begin{array}{c}\text { Centroid } \\
{\left[\mathrm{km} \mathrm{s}^{-1}\right]}\end{array}$ & $\begin{array}{c}\text { Eq. width } \\
{\left[\mathrm{km} \mathrm{s}^{-1}\right]}\end{array}$ \\
\hline $\mathrm{CO}(J=1-0)$ & 0.061 & $8.18 \pm 0.04 \pm 0.82$ & $515 \pm 1$ & $134 \pm 1$ \\
$\mathrm{CO}(J=2-1)$ & 0.046 & $6.28 \pm 0.03 \pm 0.62$ & $513 \pm 1$ & $135 \pm 1$ \\
\hline
\end{tabular}

calibration of the $\mathrm{CO}(J=1-0)$ and $\mathrm{CO}(J=2-1)$ data may be better than the absolute calibration and hence the error in their ratio can be smaller than indicated by the absolute error.

\subsection{The maps}

\subsection{1. $\mathrm{CO}(J=1-0)$}

Figure 4 shows maps of the $\operatorname{CO}(J=1-0)$ velocity-integrated intensity (hereafter $I_{1-0}$ ) and the peak intensity (hereafter $T_{1-0}$ ) for both the convolved data set (left column) and the MEM-deconvolved data set (right column).
The $\mathrm{CO}(J=1-0)$ emission is concentrated to the nucleus, the central part of the bar, the bar ends, and the spiral arms. The velocity-integrated intensity at the nucleus and the eastern and western bar ends are: 73,24 , and $30 \mathrm{~K} \mathrm{~km} \mathrm{~s}^{-1}$, respectively, which is very close to the corresponding values in Crosthwaite et al. (2002): 73, 22, and $28 \mathrm{~K} \mathrm{~km} \mathrm{~s}^{-1}$. In the MEM-deconvolved data set these features are brighter due to the increased spatial resolution: at the eastern bar end we measure $49 \mathrm{~K} \mathrm{~km} \mathrm{~s}^{-1}$, at the western bar end $52 \mathrm{~K} \mathrm{~km} \mathrm{~s}^{-1}$, and at the nucleus the maximum $I_{1-0}$ is $235 \mathrm{~K} \mathrm{~km} \mathrm{~s}^{-1}\left(T_{1-0}=2.8 \mathrm{~K}\right)$. By fitting an ellipse to the MEM-deconvolved $I_{1-0}$-distribution we find that the CO peak coincides with the IR nucleus within the absolute position accuracy of our maps $\left(3^{\prime \prime}\right)$. The axes ratio of this ellipse is $60^{\prime \prime}: 29^{\prime \prime}$ with a position angle of $36^{\circ}$. In the MEMdeconvolved maps the spiral arms are resolved, and they can be followed for almost $360^{\circ}$. The emission in the spiral arms breaks up into regularly spaced maxima, which we interpret to be giant molecular associations (GMAs), a phenomenon previously seen in e.g. M 51 (Nakai et al. 1994). The outer arm, to the SE of the nucleus, seems somewhat disturbed in the sense that in the MEM-deconvolved $T_{1-0}$ map it is possible to discern two parallel arms. Further toward the west, this arm appear to almost merge with the inner arm, which also appears to be the case on the opposite side of the galaxy (NE of the nucleus). 


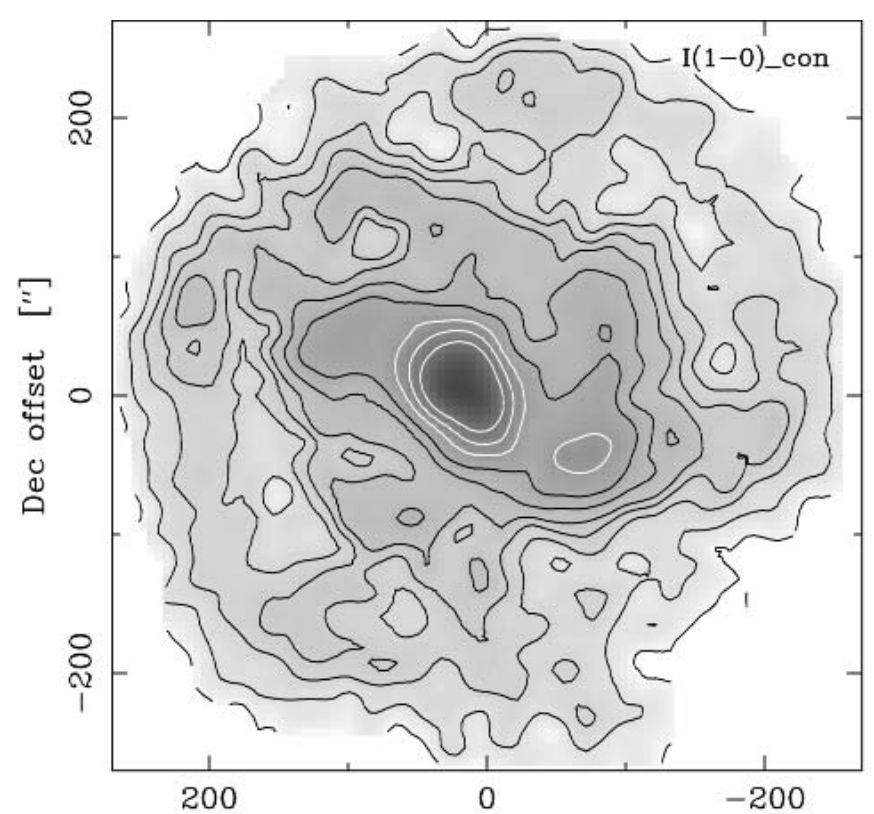

RA offset ["]

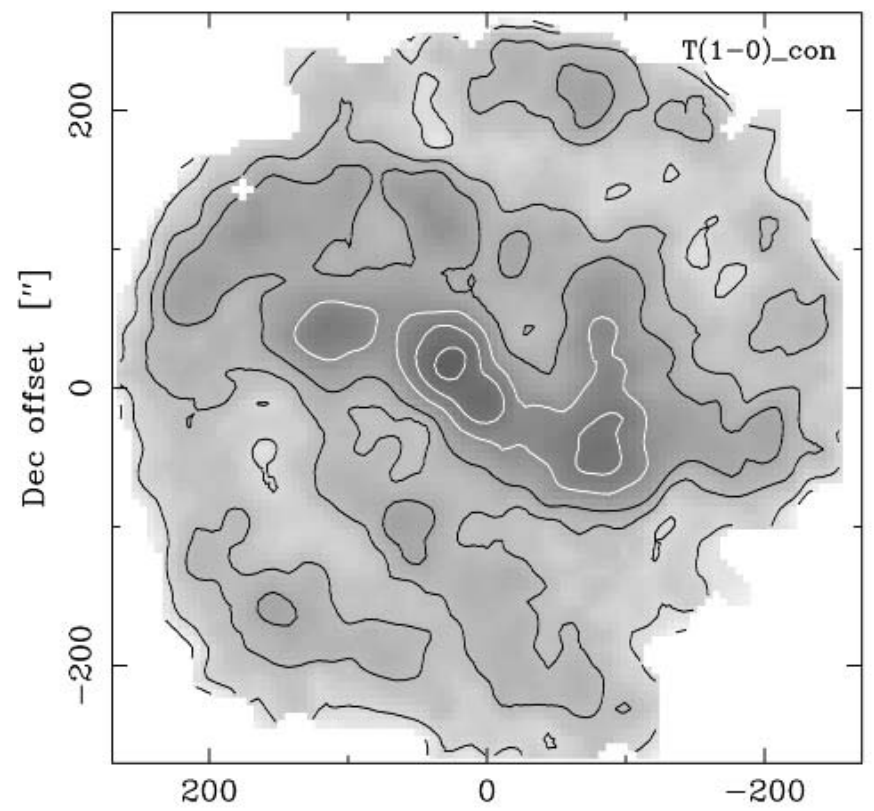

RA offset ["]

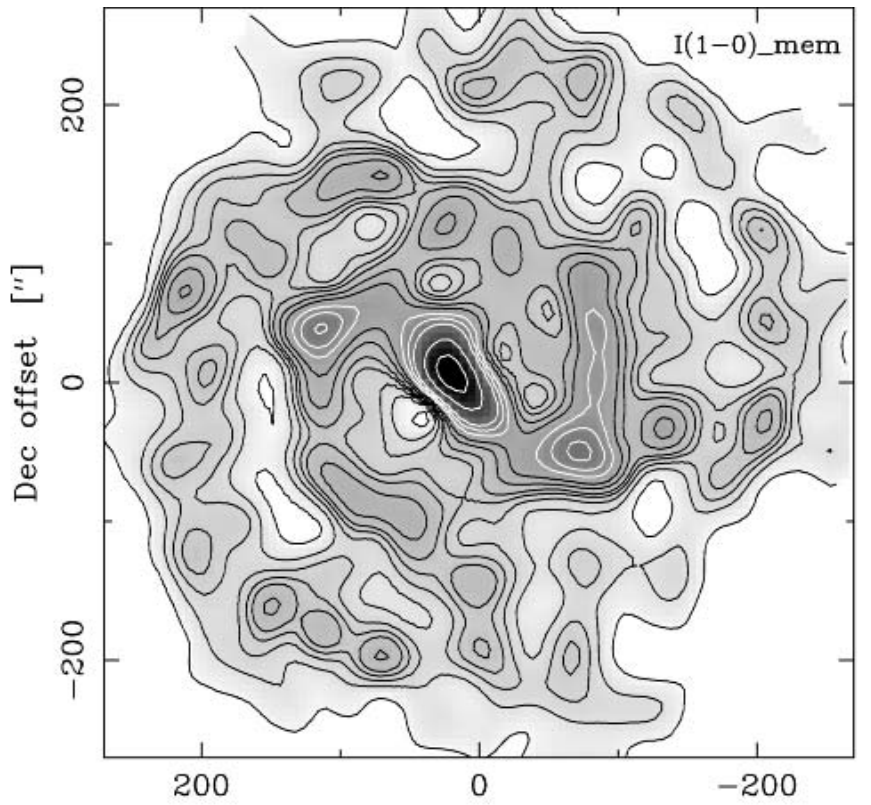

RA offset ["]

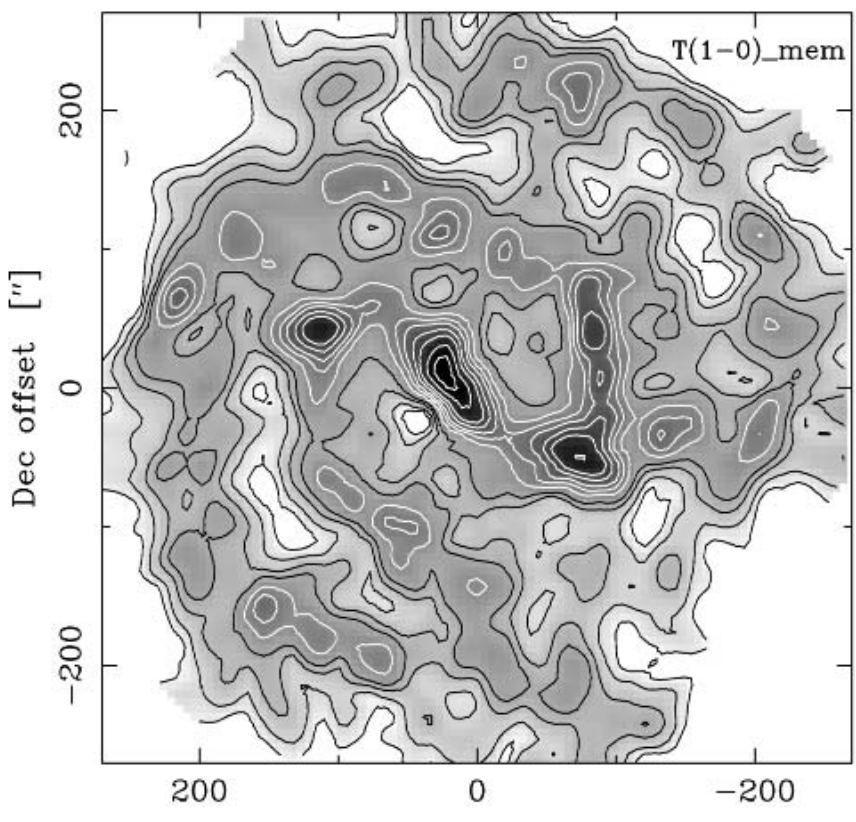

RA offset ["]

Fig. 4. The $\mathrm{CO}(J=1-0)$ maps. Velocity-integrated intensity in the convolved data set (resolution $\approx 49^{\prime \prime}$; upper left) and in the MEM-deconvolved data set (resolution $\approx 22^{\prime \prime}$; upper right). Contours are: 1, 3, 5, 7, 9, 13, 18, 27 (first white contour), 37, 50, 100 and $175 \mathrm{~K} \mathrm{~km} \mathrm{~s}^{-1}$. Maximum intensities in the convolved and MEM-deconvolved maps are 127 and $235 \mathrm{~K} \mathrm{~km} \mathrm{~s}^{-1}$, respectively. Peak intensities in the convolved data set (lower left) and in the MEM-deconvolved data set (lower right). Contours are: 0.05, 0.1, 0.2, 0.3, 0.5 (first white contour), 0.7, 0.9, 1.2, 1.5, 2.0 and $2.5 \mathrm{~K}$. Peak intensity at the center are 1.4 and $2.8 \mathrm{~K}$ for the convolved and deconvolved set, respectively.

Rand et al. (1999) (hereafter RLH) mapped the $\mathrm{CO}(J=1-0)$ emission in the inner eastern arm using the OVRO Millimeter Array (spatial resolution 6!'5 × 3.'5). In Fig. 5 we show their data overlayed on our deconvolved $I_{1-0}$ map. The components close to the nucleus (components 1-5 using their nomenclature), are not prominent in our data. They are distributed along the leading edge of the bar. The reason is probably that a shock in this region creates substructures (in temperature, in density, and/or in velocity) in the $\mathrm{CO}$ distribution which elsewhere is smooth and therefore undetectable by an interferometer (RLH found that only $2-5 \%$ of the single-dish emission was recovered in their interferometer observations). Our eastern bar end is located between components 6 and 7, and the spiral arm segment 

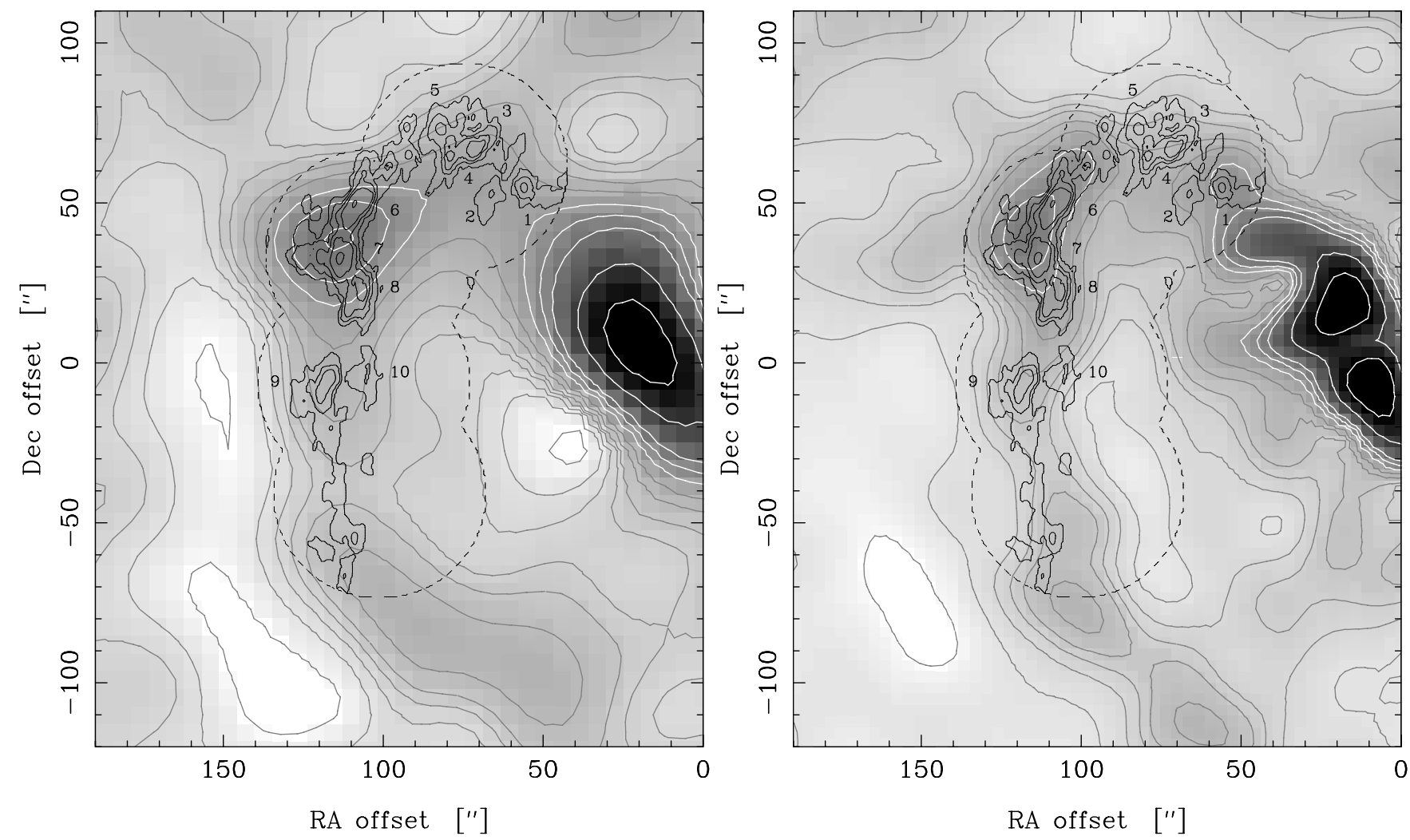

Fig. 5. OVRO interferometer $\mathrm{CO}(J=1-0)$ emission (thin lines) superposed on a gray+contour map of our $\operatorname{CO}(J=1-0)$ (left) and $\mathrm{CO}(J=2-1)$ data (right).

which continues southward is located between components 9 and 10 .

\subsection{2. $\mathrm{CO}(J=2-1)$}

The $\mathrm{CO}(J=2-1)$ maps show structures very similar to those in the $\operatorname{CO}(J=1-0)$ maps, however with a higher angular resolution, Fig. 6. The deconvolved $I_{2-1}$ map shows the "double nucleus" previously seen in the $\operatorname{CO}(J=3-2)$ and $\operatorname{CO}(J=4-3)$ lines by Petitpas \& Wilson (1998). This "double nucleus" is apparent also in our raw $\mathrm{CO}(J=2-1)$ data, but it is not possible to see in the convolved $I_{2-1}$ map, since this map is made using data which was both convolved (with a $15^{\prime \prime}$ beam) and regridded (to $11^{\prime \prime}$ spacing). Since the gray-scale and contour levels in Figs. 4 and 6 are the same, we can directly infer that the $\operatorname{CO}(J=2-1)$ emission is less concentrated to the arms than the $\mathrm{CO}(J=1-0)$ emission.

\section{Results and discussion}

\subsection{Molecular mass in $M 83$}

The $\mathrm{H}_{2}$ column density in external galaxies is commonly derived from the velocity-integrated $\operatorname{CO}(J=1-0)$ intensity $\left(I_{\mathrm{CO}}=\int T_{\mathrm{mb}} \mathrm{d} v\right)$ using the expression

$N\left(\mathrm{H}_{2}\right)=X_{\mathrm{CO}} \times I_{\mathrm{CO}}$,

where $X_{\mathrm{CO}}$ is a conversion factor. The actual value of this factor, and its applicability in different environments have been widely discussed, see e.g. Wilson (1995), Arimoto et al. (1996), Combes (2000), Young \& Rownd (2001). In this paper we have adopted the value $X_{\mathrm{CO}}=2.3 \times 10^{20}\left(\mathrm{~K} \mathrm{~km} \mathrm{~s}^{-1}\right)^{-1} \mathrm{~cm}^{-2}$ (Strong et al. 1988), which is supported by the analysis done in RLH. In order to convert the measured intensities into mass surface density we used the expression

$\Sigma\left(\mathrm{H}_{2}\right)=3.7 \times I_{\mathrm{CO}} \times \cos i=3.3 \times I_{\mathrm{CO}} M_{\odot} \mathrm{pc}^{-2}$,

where $i$ is the inclination of the galaxy, and $I_{\mathrm{CO}}$ is given in $\mathrm{K} \mathrm{km} \mathrm{s}^{-1}$ (This expression can be obtained by integrating Eq. (1)). Using this conversion factor to convert the integrated intensity of the global $\mathrm{CO}(J=1-0)$ spectrum $\left(8.18 \mathrm{~K} \mathrm{~km} \mathrm{~s}^{-1}\right.$, Table 4) we find that the average (over the entire optical disk, and including the bar and the nucleus) surface mass density is $27.2 M_{\odot} \mathrm{pc}^{-2}$, and that the total molecular gas mass within the surveyed area is $3.3 \times 10^{9} M_{\odot}$.

\subsection{The radial distribution}

\subsubsection{Molecular gas}

We have, using the sliding-window technique described above, calculated the velocity-integrated intensity, centroid velocity, peak intensity, and center and width of a Gaussian emission profile fitted to the spectrum for all spectra in all data cubes. The left panel of Fig. 7 shows the $\operatorname{CO}(J=1-0)$ velocityintegrated intensities (obtained from the convolved data set) as a function of the (inclination-corrected) distance from the galaxy center. The scatter is dominated by the arm-interarm 


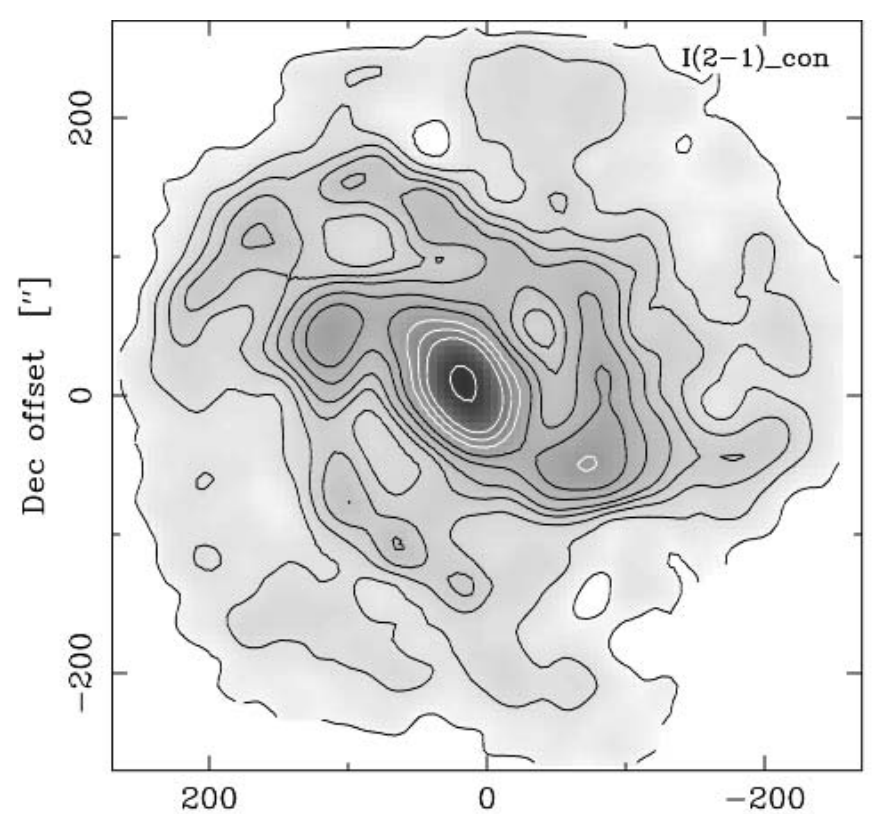

RA offset ["]

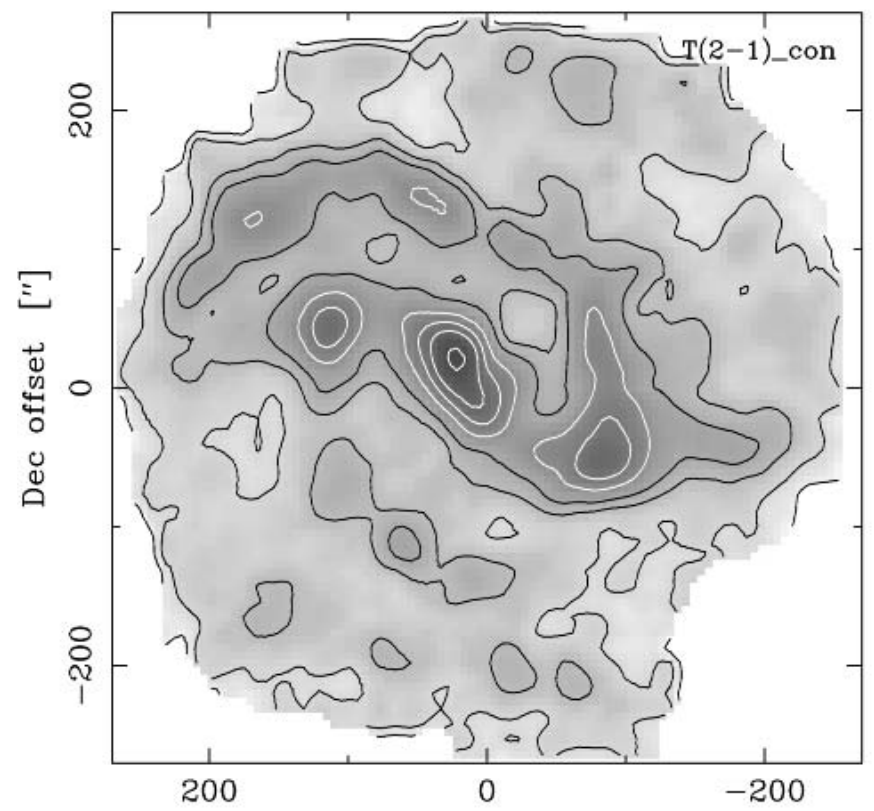

RA offset ["]

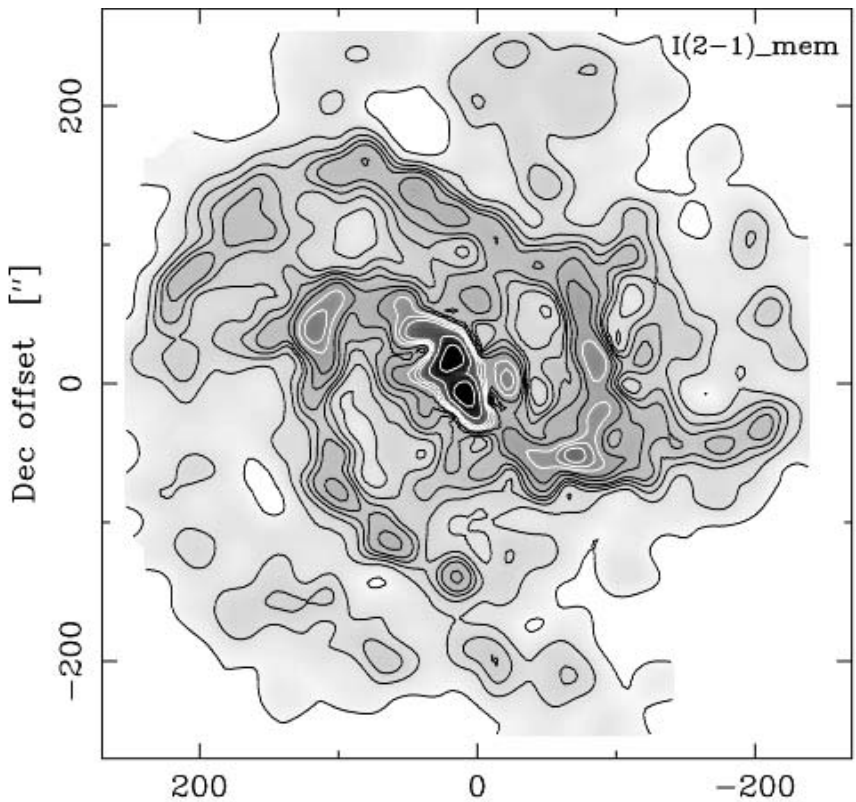

RA offset ["]

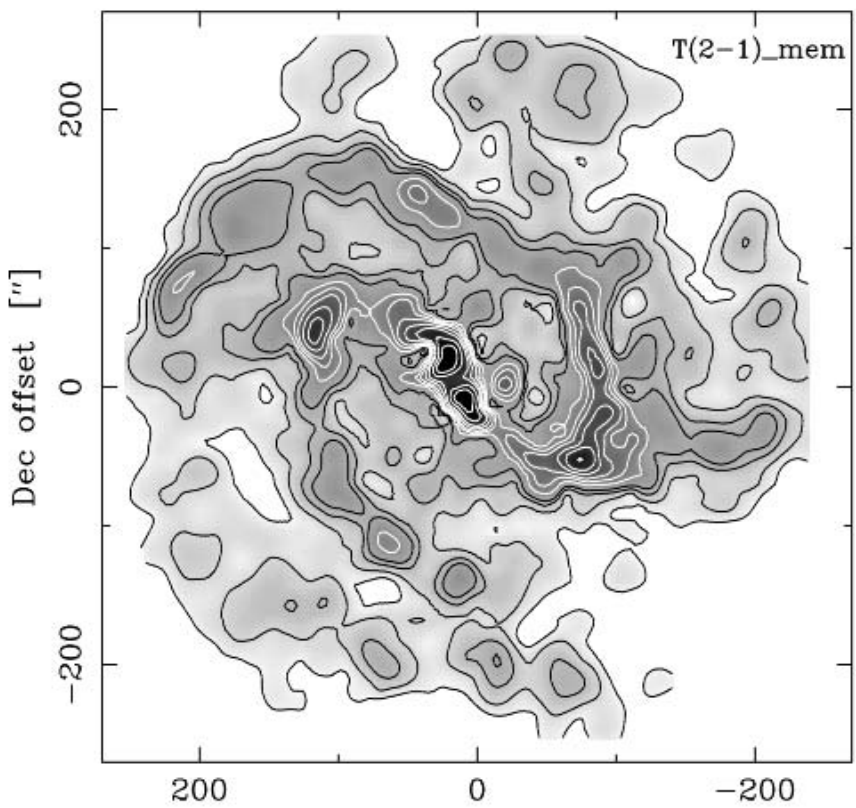

RA offset ["]

Fig. 6. Same as in Fig. 4 but for $\mathrm{CO}(J=2-1)$. The contours are also the same. The resolution of the maps are $27^{\prime \prime}$ and $12^{\prime \prime}$ for the convolved and the MEM-deconvolved data set, respectively. For the convolved data maps the values at the center are $110 \mathrm{~K} \mathrm{~km} \mathrm{~s}^{-1}$ and $1.3 \mathrm{~K}$. In the MEM-deconvolved map, the nucleus breaks up into two discrete sources, with similar velocity-integrated and peak intensities: $240 \mathrm{~K} \mathrm{~km} \mathrm{~s}^{-1}$ and $3.0 \mathrm{~K}$.

contrast. The data in the right panel of Fig. 7 were obtained from the integrated intensities of spectra averaged within concentric annuli (see Fig. 3 and Table 5) by converting the $\mathrm{CO}(J=1-0)$ integrated intensity into mass surface density as described above. In this procedure we used the raw data set, since we needed neither the higher spatial resolution of the MEM-deconvolved spectra nor the lower noise of the convolved spectra. The error bars reflect the uncertainty in the azimuthally-averaged radial distribution. To these data we fitted an $\mathrm{H}_{2}$ mass surface density distribution which is a combination of a Gaussian component and an exponential function (for the disk),

$$
\begin{aligned}
\Sigma\left(\mathrm{H}_{2}\right)= & 216 \exp \left[-\left(\frac{r}{649 \mathrm{pc}}\right)^{2}\right] \\
& +120 \exp \left[-\frac{r}{2265 \mathrm{pc}}\right] M_{\odot} \mathrm{pc}^{-2} .
\end{aligned}
$$



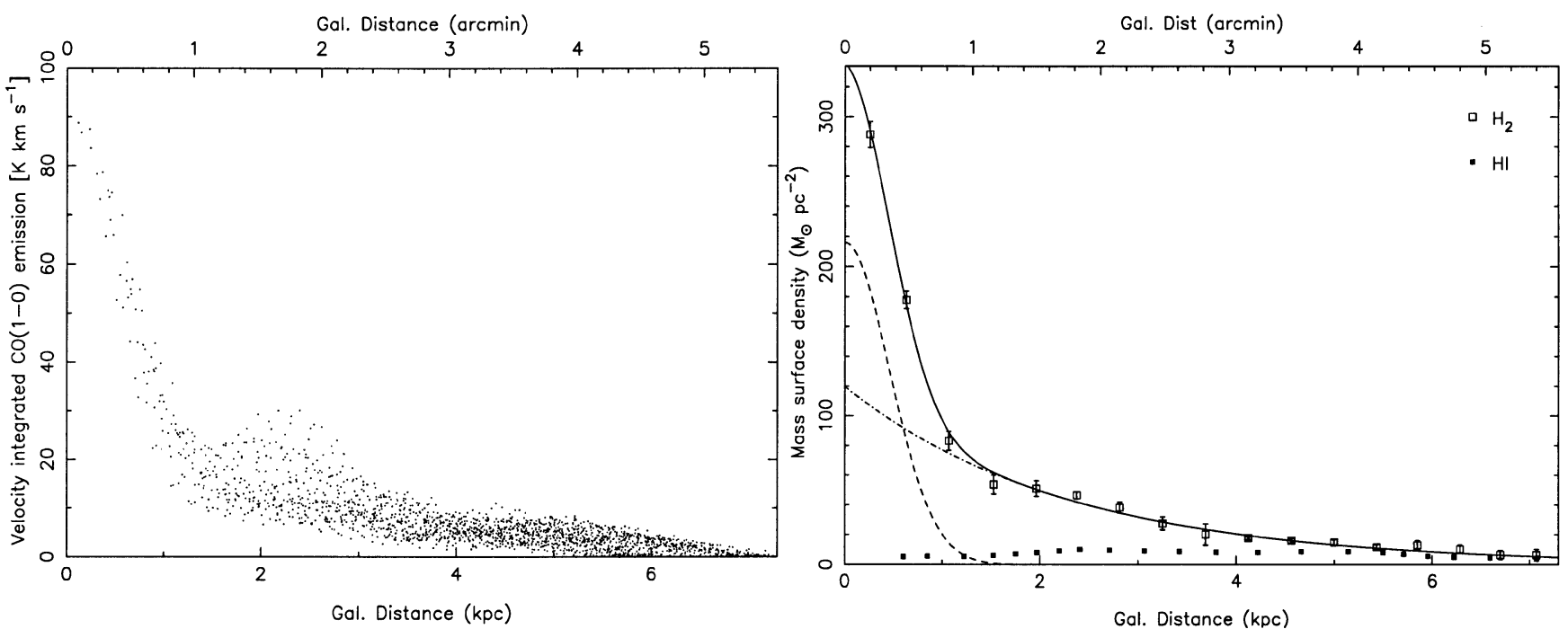

Fig. 7. The $\mathrm{CO}(J=1-0)$ velocity-integrated intensity versus galactocentric distance is shown in the left panel (obtained from the convolved data set). The dispersion is dominated by the arm-interarm contrast, and the bar-ends are evident at $\approx 2 \mathrm{kpc}$. In the right panel the $\mathrm{H}_{2}$ mass surface densities are shown with formal errors (the data are taken from the spectra in Fig. 3), together with HI mass surface densities. Also shown, as a solid line, is the fit of an $\mathrm{H}_{2}$ mass distribution described in the text. It consists of a Gaussian central part (dashed line) and an exponential disk (dash-dotted line).

Table 5. $\mathrm{CO}(J=1-0)$ and $\mathrm{CO}(J=2-1)$ velocity-integrated intensities of spectra averaged within inclination-corrected annuli. The annuli are fully sampled out to $230^{\prime \prime}$. The $\mathrm{CO}(J=2-1)$ data were regridded to the same spacing as the $\operatorname{CO}(J=1-0)$ so that the disk was sampled in the same way for both transitions. The difference in angular resolution in the data sets has an effect on the response at the center. The errors $(5 \sigma)$ do not include the calibration uncertainty.

\begin{tabular}{lccccc}
\hline \hline $\begin{array}{l}\text { radius } \\
{\left[{ }^{\prime}\right]}\end{array}$ & $\begin{array}{c}I_{1-0} \\
{\left[\mathrm{~K} \mathrm{~km} \mathrm{~s}^{-1}\right]}\end{array}$ & $\begin{array}{c}\text { error } \\
{\left[\mathrm{K} \mathrm{km} \mathrm{s}^{-1}\right]}\end{array}$ & $\begin{array}{c}I_{2-1} \\
{\left[\mathrm{~K} \mathrm{~km} \mathrm{~s}^{-1}\right]}\end{array}$ & $\begin{array}{c}\text { error } \\
{\left[\mathrm{K} \mathrm{km} \mathrm{s}^{-1}\right]}\end{array}$ & $\begin{array}{c}\text { \#spectra } \\
\text { in annuli }\end{array}$ \\
\hline 12 & 87.7 & 2.1 & 98.1 & 0.8 & 9 \\
29 & 53.0 & 1.2 & 51.6 & 0.5 & 27 \\
49 & 25.4 & 1.8 & 19.1 & 0.7 & 49 \\
70 & 15.5 & 1.6 & 10.2 & 0.7 & 67 \\
90 & 15.6 & 1.6 & 11.0 & 0.4 & 85 \\
109 & 14.2 & 0.8 & 11.5 & 0.7 & 100 \\
129 & 11.7 & 0.9 & 8.2 & 0.4 & 126 \\
149 & 7.9 & 1.1 & 5.7 & 0.8 & 141 \\
169 & 6.3 & 1.2 & 4.3 & 0.6 & 157 \\
189 & 5.4 & 0.2 & 3.6 & 0.8 & 181 \\
209 & 4.8 & 0.5 & 3.6 & 0.5 & 198 \\
229 & 4.4 & 0.5 & 3.1 & 0.6 & 215 \\
249 & 3.5 & 0.4 & 2.4 & 0.7 & 212 \\
268 & 3.7 & 0.8 & 2.1 & 0.6 & 155 \\
288 & 3.0 & 0.7 & 1.5 & 0.6 & 102 \\
307 & 2.1 & 0.9 & $<2.7$ & & 44 \\
\hline
\end{tabular}

The Gaussian shape of the inner part of the distribution depends mainly on the convolution of the true distribution with the beam (the scale length 649 pc in Eq. (3) corresponds to a $F W H M$ of $\left.50^{\prime \prime}\right)$. The right panel of Fig. 7 shows also the mass surface density of the HI gas. These data were obtained from Tilanus \& Allen (1993) (hereafter TA). The column densities in TA were obtained with the VLA, and the authors noted that their estimated total HI mass within the Holmberg radius $\left(0.87 \times 10^{9} M_{\odot}\right)$ was lower than the corresponding masses estimated by other authors using single dish observations, e.g., both Rogstad et al. (1974) and Huchtmeier \& Bohnenstengel (1981) obtained about $1.6 \times 10^{9} M_{\odot}$ (all masses 
recalculated to the distance $4.5 \mathrm{Mpc}$ ). The most probable explanation for the deficiency was, according to TA, the absence of short-spacing information. In order to compensate for this we have added the "missing" mass surface density $\left(2.4 M_{\odot} \mathrm{pc}^{-2}\right)$ to the HI data of TA. The resulting HI mass surface densities are still low and typically $\mathrm{H}_{2}$ gas constitute about $70 \%$ of the total mass of neutral hydrogen $\left(\mathrm{H}_{2}+\mathrm{HI}\right)$ in the disk inside $6.8 \mathrm{kpc}$.

Based upon the $\mathrm{H}_{2}$ mass surface density distribution (Eq. (3)) we estimate a total molecular gas mass within a radius of $7.3 \mathrm{kpc}$ (the extent of the map) of $3.5 \times 10^{9} M_{\odot}$, and extrapolated to the Holmberg radius $(7 ! 3$ or $9.6 \mathrm{kpc})$ the mass becomes $3.9 \times 10^{9} M_{\odot}$ (which agrees well with the result of Crosthwaite et al. (2002), $3.7 \times 10^{9} M_{\odot}$ (cf. also to the result $3.3 \times 10^{9} M_{\odot}$ that was derived in Sect. 5.1 using the velocity-integrated intensity in the global spectrum). This is more than twice the total HI mass within the same galactocentric radius. It should, however, be noted that $80 \%$ of the HI mass in M 83 is found outside the Holmberg radius (Huchtmeier \& Bohnenstengel 1981). As a comparison, the stellar mass of M 83 is $\sim 37 \times 10^{9} M_{\odot}$, given $V_{\mathrm{T}}^{0}=7.37$ (de Vaucouleurs et al. 1992) and assuming a mass-to-light ratio of 2 .

In the Gaussian/exponential disk decomposition the major part of the molecular gas mass within the Holmberg radius lies in the disk, $3.6 \times 10^{9} M_{\odot}$. Lord (1987) made $\operatorname{CO}(J=1-0)$ observations in 21 positions in the central parts of $\mathrm{M} 83$, and he estimated the total molecular gas mass within $115^{\prime \prime}$ to be $1.2 \times 10^{9} M_{\odot}$ (we have compensated for the difference in $X_{\mathrm{CO}}$ and distance). Our $\mathrm{H}_{2}$ distribution gives $1.4 \times 10^{9} M_{\odot}$.

\subsubsection{Comparison with data at other wavelengths}

In Fig. 8 we compare the $\mathrm{CO}$ radial distributions with corresponding photometry data in the $B, K$, and $\mathrm{H} \alpha$ bands. The $B$ and $\mathrm{H} \alpha$ data come from Talbot et al. (1979), and the $K$ data from Adamson et al. (1987). The scale lengths of the various tracers compare well over most of the radial range, but the comparatively low spatial resolution affects the $\mathrm{CO}$ curves in the central region and at the bar ends. The latter are responsible for the peaks at $\sim 120^{\prime \prime}$. We have fitted a single exponential to the data in the radial range $140^{\prime \prime}-240^{\prime \prime}$, which corresponds to the region outside the bar but inside the area where our $\mathrm{CO}$ survey is complete. The scale lengths are: $135 \pm 14^{\prime \prime}, 126 \pm 16^{\prime \prime}$, $112 \pm 20^{\prime \prime}, 100 \pm 14^{\prime \prime}$ and $132 \pm 35^{\prime \prime}$ for $\operatorname{CO}(J=1-0)$, $\mathrm{CO}(J=2-1), K, B$, and $\mathrm{H} \alpha$, respectively (the errors of the latter three do not include the measurement errors). Thus, the disk scale lengths are, within the errors, the same for these star formation tracers.

The CO intensities decrease exponentially over the entire surveyed area, and we do not observe the sharp cut-off at 4.5 seen by Crosthwaite et al. (2002). In fact, we find CO emission with $I_{1-0} \approx 1 \mathrm{~K} \mathrm{~km} \mathrm{~s}^{-1}$ in selected spectra as far from the nucleus as 5.5.

\subsection{Brightness distributions}

We find that in the MEM-deconvolved map the average $\mathrm{CO}(J=1-0)$ intensity in the arms is about 2.5 times higher

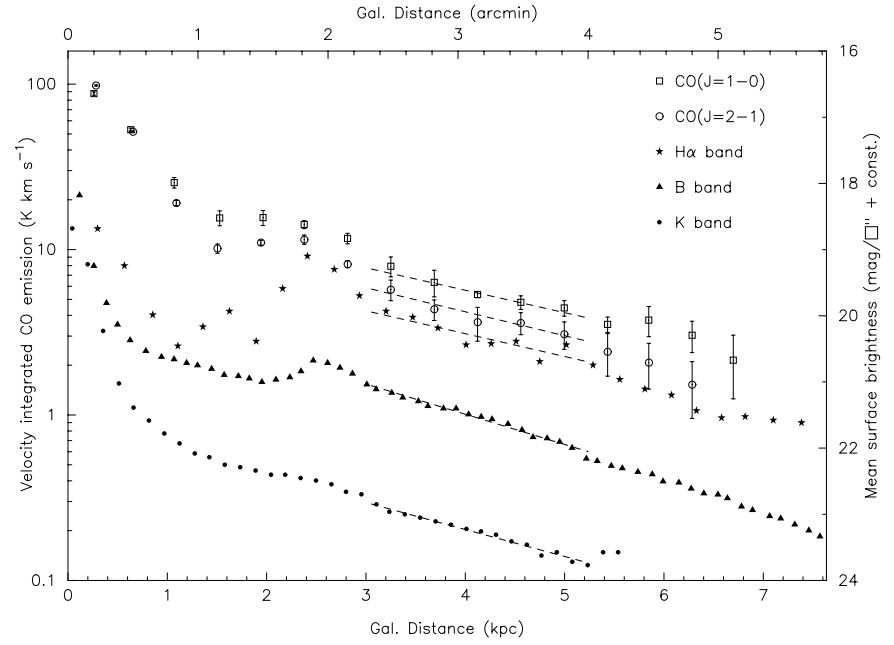

Fig. 8. Azimuthally-averaged $\mathrm{CO}(J=1-0)$ and $\mathrm{CO}(J=2-1)$ velocity-integrated intensity (cf. Table 5) in annular zones compared to surface brightnesses in the $\mathrm{H} \alpha, B$, and $K$ filters. Note how the bar ends appear as a bump at $120^{\prime \prime}$ in the distributions. The spatial resolution of the $\operatorname{CO}(J=2-1)$ is better than that of the $\operatorname{CO}(J=1-0)$ data, which results in the difference in slopes of the inner parts of these distributions. The dashed lines show the fits of exponentials discussed in the text.

than in the interarm regions. Assuming a direct proportionality between $\mathrm{CO}$ emission and mass surface density, this agrees well with the lower limit of the arm-interarm mass surface density ratio, 2.3 , found by RLH using purely kinematical arguments.

In Fig. 9 we show the MEM-deconvolved $\operatorname{CO}(J=1-0)$ velocity-integrated intensity map superposed on a true-color image. This image is a composite of three images in the filters $B, V$, and $R$, rendered as blue, green, and red, respectively. These images have been obtained at the ESO Danish $1.54 \mathrm{~m}$ telescope by Sören Larsen and the data are described in Larsen \& Richtler (1999). On the large scale the $\operatorname{CO}(J=1-0)$ emission follows the dust lanes, both in and between spiral arms, but at some locations the $\operatorname{CO}(J=1-0)$ emission is displaced toward the regions where star formation takes place (Wiklind et al. 1990). Most notably, in the inner spiral arm on the eastern side, the $\mathrm{CO}(J=1-0)$ ridge is located downstream of the dust lane with a separation of about 10-15" (also shown in $\mathrm{RLH}$ ), as are the $\mathrm{HI}$ and $\mathrm{H} \beta$ emissions as shown by TA. We note that in general the OB associations straddle the rims of the $\operatorname{CO}(J=1-0)$ emission concentrations, presumably due to extinction in combination with the photodissociation by the young stellar population. The outer and inner spiral arms appear to connect at several locations, and these connection regions often show an increased number of $\mathrm{OB}$ associations. The "Gould belt structure", at $(1.75,-2 ! 25)$ in the coordinate system of Fig. 9, is such a region (Comerón 2001).

Figure 10 shows the $\operatorname{CO}(J=2-1)$ velocity-integrated intensity as contours on an RGB image, where the colors represent star formation $(\mathrm{H} \alpha$; red), infrared emission ( $I$ band; green), and dust lanes (filters $V-I$; blue). In the disk, the observed $\operatorname{CO}(J=2-1)$ brightness distribution strengthens the conclusions drawn from the $\operatorname{CO}(J=1-0)$ emission. 


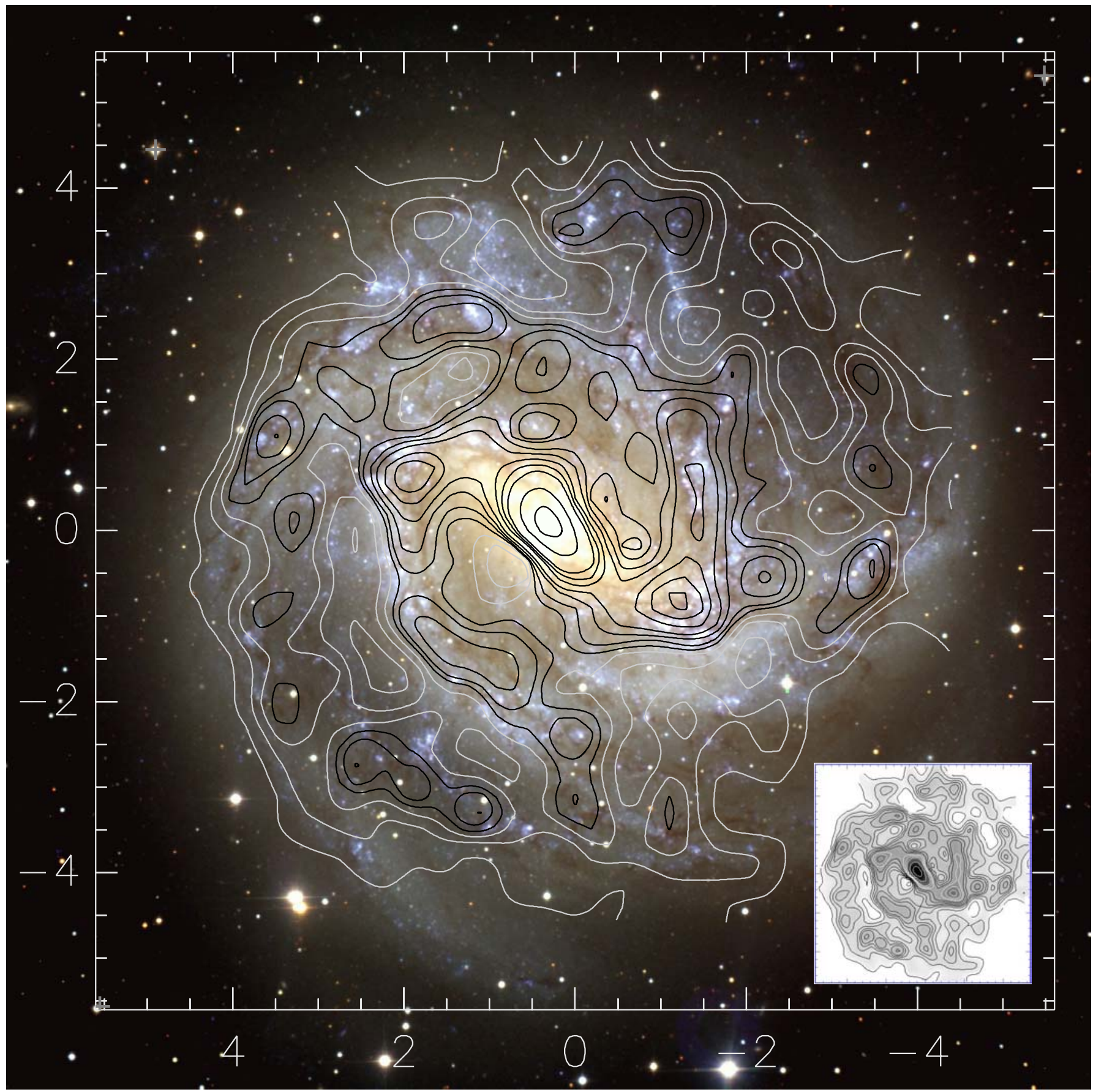

Fig. 9. The MEM-deconvolved velocity-integrated $\mathrm{CO}(J=1-0)$ intensity as contours superposed on an RGB-map produced from images in $B$, $V$, and $R$. The optical images were obtained by Sören Larsen at the ESO Danish $1.5 \mathrm{~m}$ telescope on La Silla, Chile. Three stars have been used for image alignment. These are located in the lower left corner and in both upper corners and are marked with crosses. The inset shows the $\mathrm{CO}(J=1-0)$ map in gray-scale.

In the central region the higher angular resolution at the $\mathrm{CO}(J=2-1)$ frequency reveals additional details. There are two central nuclear components relatively symmetrically placed on opposite sides of the IR center. At these locations the dust lanes, on the leading edges of the bar, attach to the nuclear ring seen in $J-K$ by Elmegreen et al. (1998). Their color difference map $(V-I)$ shows an area of high dust extinction at the location of the NE component, whereas at the SW component there appears to be relatively small amounts of dust.
It appears as if the NE CO component lies above the disk (or the bulge) and the SW CO component lies below or behind the same. This was also concluded by Sofue \& Wakamatsu (1994) based on similar data. Along the bar, the $\mathrm{CO}(J=2-1)$ emission follows the dust lanes on the leading edges. In the disk the $\mathrm{CO}$ emission traces the $\mathrm{H} \alpha$ emission very well, although there are regions with a clear anti-correlation, i.e., substantial $\mathrm{CO}$ emission but none, or very weak, $\mathrm{H} \alpha$ emission, such as the peak at about half an arcminute west of the nucleus. 


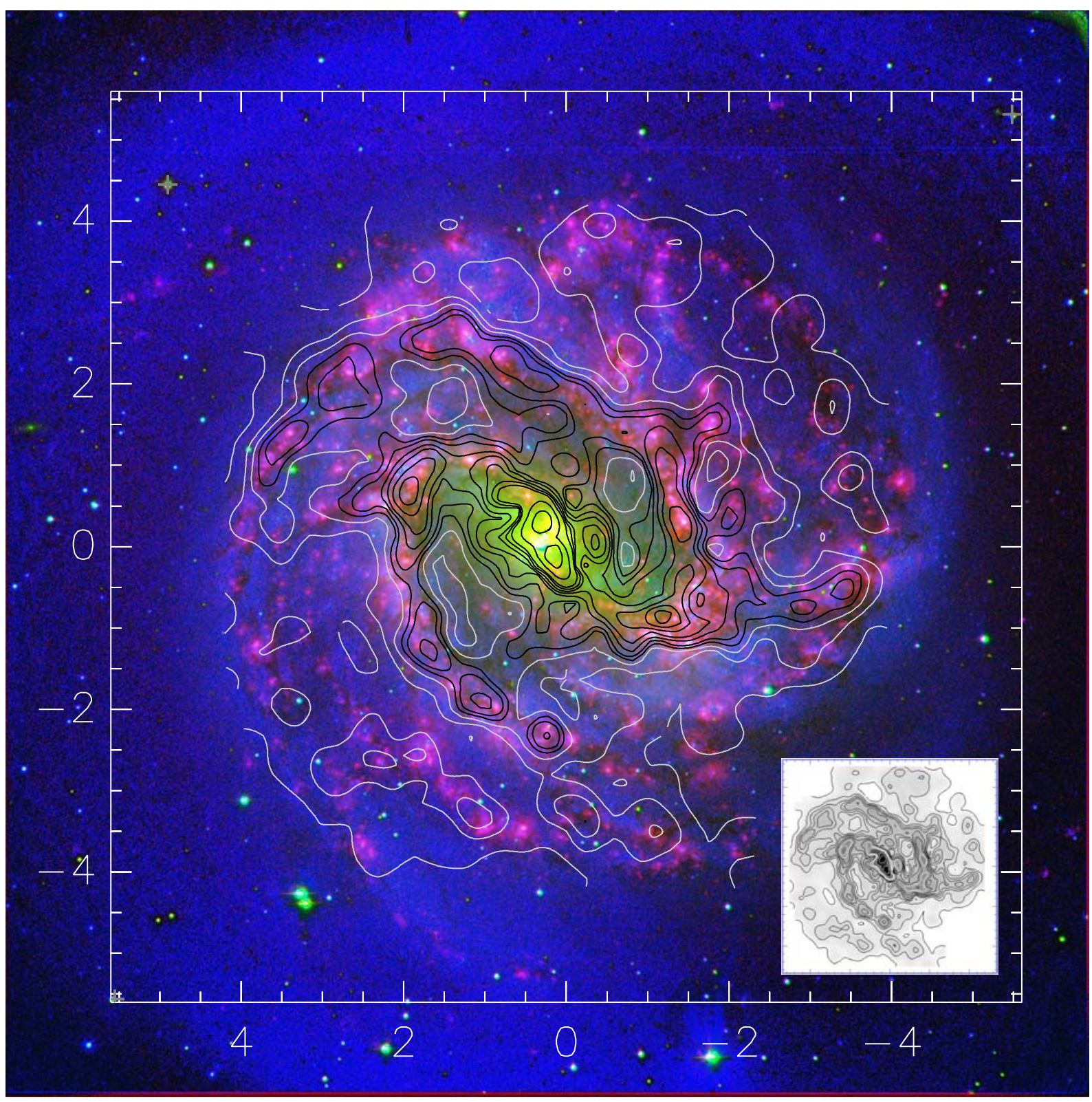

Fig. 10. The MEM-deconvolved $\mathrm{CO}(J=2-1)$ velocity-integrated intensity as contours on an RGB image where the colors red, green and blue represent star formation $(\mathrm{H} \alpha)$, infrared emission ( $I$ band), and dust lanes (filters $V-I)$, respectively. The inset shows the $\mathrm{CO}(J=2-1)$ map in gray-scale.

Figure 11 shows the $\operatorname{CO}(J=1-0)$ velocity-integrated intensity on a gray-scale image of the HI column density (data kindly provided by R. P. J. Tilanus and R. J. Allen). The angular resolution of the HI map is slightly better than that of the CO map. The CO emission (which is assumed to be linearly proportional to the $\mathrm{H}_{2}$ mass surface density intensity) and the HI column density follow each other closely, with one outstanding exception: the nucleus, where very little $\mathrm{HI}$ is present. At some places in the disk the $\mathrm{H}_{2}$ and $\mathrm{HI}$ maxima are displaced from each other, but there is no apparent systematic trend that $\mathrm{H}_{2}$ is outside $\mathrm{HI}$ or vice versa. In general, the separations are of the order of $3-9^{\prime \prime}$, which is small compared to the resolution in these maps $\left(23^{\prime \prime}\right)$.

Local minima in the $\mathrm{HI}$ and $\mathrm{H}_{2}$ gas surface densities also correlate well. At $\left(50^{\prime \prime}, 180^{\prime \prime}\right)$ in the coordinate system of
Fig. 11, marked minima can be seen in both $\mathrm{H}_{2}$ and HI. The total gas surface density in this area is about $3-6 M_{\odot} \mathrm{pc}^{-2}$, of which only $\approx 1 M_{\odot} \mathrm{pc}^{-2}$ is in the form of molecular gas. Such low mass surface densities should not give rise to any massive star formation, and indeed, the $\mathrm{H} \alpha$ image shows very little emission here.

Figure 12 compares the $\mathrm{H}_{2}$ (as probed by the $\mathrm{CO}(J=2-1)$ emission) and the $\mathrm{HI}$ gas in more detail, the resolution being $\approx 10^{\prime \prime}$. In the main disk the $\operatorname{CO}(J=2-1)$ emission and HI emission follow each other closely and several of the concentrations in the $\operatorname{CO}(J=2-1)$ emission are also seen in the HI distribution. Again the exception is the nuclear region. The mass surface density of HI at the two nuclear components differ markedly: the SW component has a peak mass surface density of $6 M_{\odot} \mathrm{pc}^{-2}$, while at the center of the NE 


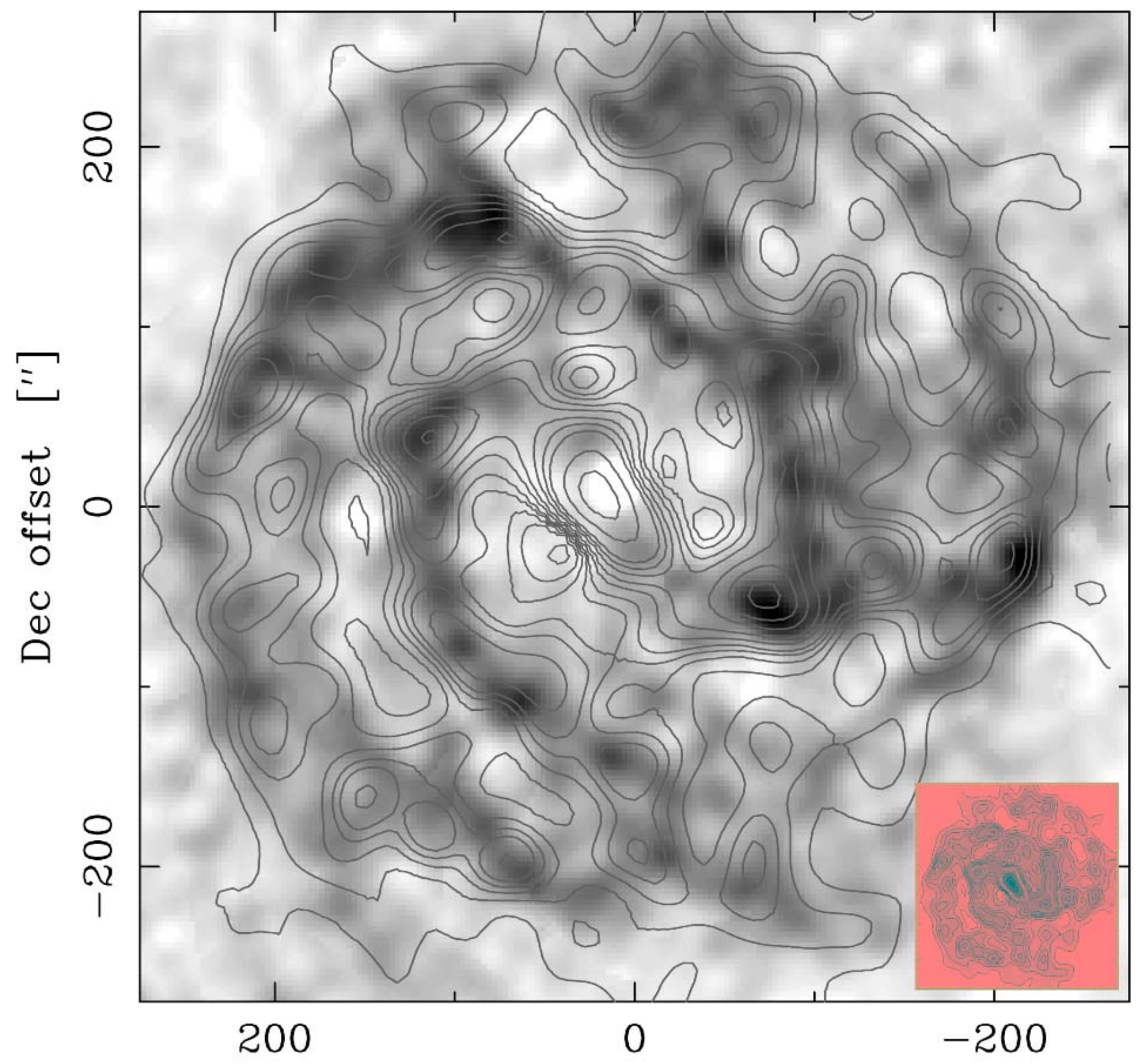

\section{RA offset [']}

Fig. 11. The MEM-deconvolved velocity-integrated $\mathrm{CO}(J=1-0)$ intensity as contours on an HI column density map (gray-scale) of roughly similar resolution $\left(\approx 20^{\prime \prime}\right)$. The HI data comes from TA. The inset shows the $\mathrm{CO}(J=1-0)$ map in gray-scale.

component $\mathrm{HI}$ is actually seen in absorption against a nuclear continuum source, which again speaks in favor of the idea that the SW and NE components lie on different sides of the disk (or a bulge). The mass surface density of $\mathrm{HI}$ at the center is very much lower than that of $\mathrm{H}_{2}$, where the highest value in the $I_{1-0}$ map corresponds to $\approx 750 M_{\odot} \mathrm{pc}^{-2}$. In the $I_{1-0}$ map it is not possible to distinguish the separate nuclei due to the relatively low spatial resolution and the coarse grid. However, using the average 2-1/1-0 line ratio (see Sect. 5.4), the mass surface density is $\sim 1100 M_{\odot} \mathrm{pc}^{-2}$ for each of these components. As the physical properties of the molecular gas in the center may differ from that in the disk, application of the standard conversion factor may lead to an erroneous result. Indeed, it is likely that at least part of the emission in the center comes from a diffuse and non-virialized component. This would lead to an overestimate of the molecular gas mass (Papadopoulos \& Allen 2000).

The explanation for the nice correlation may be that the HI is mostly a dissociation product, as TA discuss. A smooth distribution of "primordial" $\mathrm{HI}$ gas would not be detected in the interferometer observations (in which roughly $50 \%$ of the HI gas 


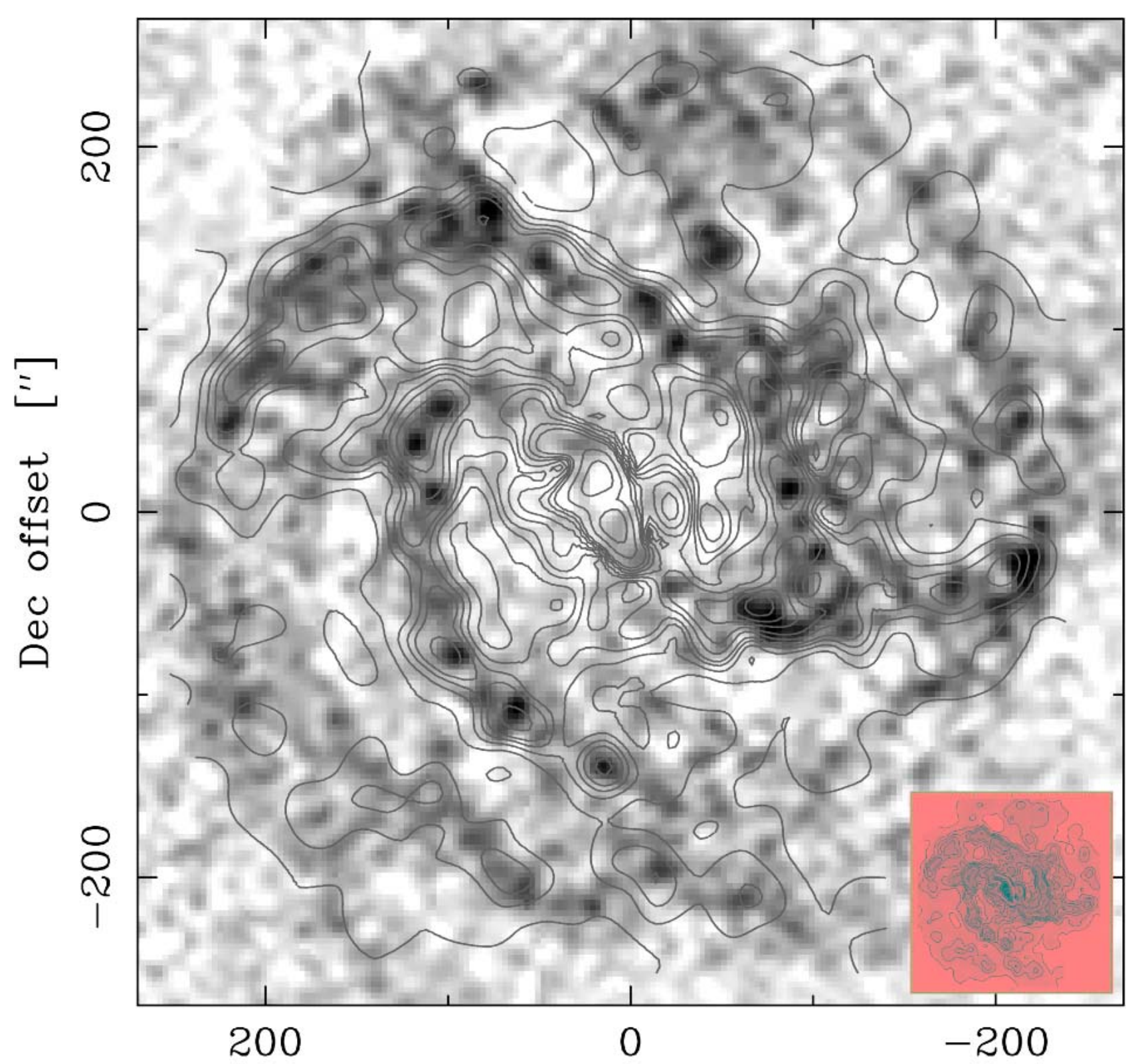

\section{RA offset ["]}

Fig. 12. The MEM-deconvolved velocity-integrated $\mathrm{CO}(J=2-1)$ intensity as contours on an HI column density map (gray-scale) of roughly similar resolution $\left(\approx 12^{\prime \prime}\right)$. The HI data comes from TA. The inset shows the $\mathrm{CO}(J=2-1)$ map in gray-scale.

was detected) while HI produced as an effect of dissociation of molecular gas in the neighborhood of star formation would be localized, and therefore detected by the interferometer.

\subsection{The $C O(J=2-1) /(J=1-0)$ line intensity ratio}

We have created a map of the $\operatorname{CO}(J=2-1) /(J=1-0)$ line intensity ratio, $R_{21}$, at $49^{\prime \prime}$ resolution. The mean value over this map is $0.72 \pm 0.19$, which is within the errors the value derived from the velocity-integrated intensities in Table 4. Crosthwaite et al. (2002) estimate a ratio of $1.1 \pm 0.2$, which we suspect is higher than ours because they have not corrected their $\mathrm{CO}(J=2-1)$ data for the error beam contribution (considering the main beam efficiency of the NRAO $12 \mathrm{~m}$ telescope at this frequency, $\approx 0.56$, the error beam is likely to be comparable to that of the SEST). In the nuclear region and along the bar the ratio is $0.83 \pm 0.04$. The $R_{21}$ map shows also that on the arms, the line intensity ratio is lower than in the interarm regions. This is illustrated in Fig. 13, where $R_{21}$ is plotted as a function of radius in a $45^{\circ}$ wide sector of the galaxy (position angle $90^{\circ}-$ $135^{\circ}$ ). In order to quantify and compare the ratio on the arms to that in the interarm regions, we have selected two sectors where the arms are well separated. The position angle of these sectors are: $90^{\circ}-135^{\circ}(\mathrm{SE})$ and $270^{\circ}-315^{\circ}(\mathrm{NW})$. In these sectors we selected spectra belonging to the inner, outer and inter-arm regions, and averaged them separately. Finally, "arm"-spectra 


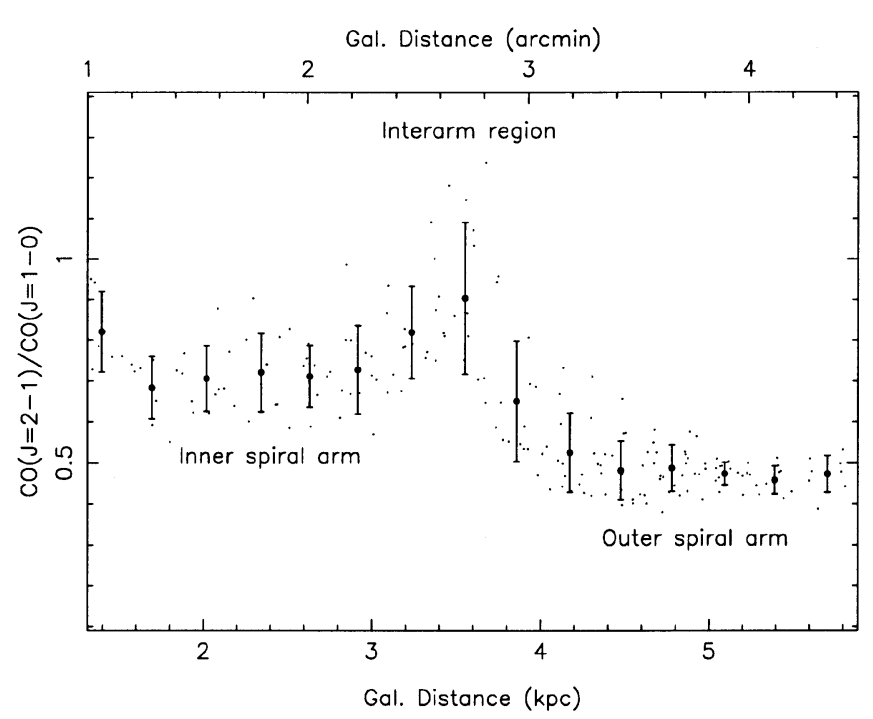

Fig. 13. The CO $(J=2-1) /(J=1-0)$ line intensity ratio, $R_{21}$, as a function of galactocentric radius. The interarm region shows a clear trend of increased line ratio. The data are taken from a $45^{\circ}$ wide sector $\mathrm{SE}$ of the nucleus (position angle $90^{\circ}-135^{\circ}$ ).

were created by taking the average of the inner and outer arm spectra in the two respective sectors. From these eight spectra, four ratios were calculated. Assuming our error beam is correct (Sect. A), the line ratio is significantly lower on the arms: $0.59 \pm 0.04(\mathrm{SE})$ and $0.69 \pm 0.04(\mathrm{NW})$ compared to the ratio in the interarm region $0.88 \pm 0.17(\mathrm{SE})$ and $0.98 \pm 0.21(\mathrm{NW})$ (the errors are $3 \sigma$ and are estimated from the noise level in the spectra). We believe that this is an effect of a (partly) different cloud component in the interarm regions, where the heating of the gas is more efficient than in the dense arm cloud complexes. As a note: We have also calculated the $R_{21}$ value for the MEM-deconvolved $\mathrm{CO}(J=1-0)$ and $\mathrm{CO}(J=2-1)$ maps at the common resolution $30^{\prime \prime}$. The arm value is roughly the same $(0.6 \pm 0.1)$, but the interarm value is higher, $1.3 \pm 0.3$.

By averaging $R_{21}$ in concentric annuli, we find a trend in the line intensity ratio in the sense that it decreases with galactocentric distance, Fig. 14 (this is also indicated in Fig. 8). In particular, the lower envelope of the $R_{21}$-values is clearly decreasing. A similar difference between the $R_{21}$ line ratio in the disk and towards the nucleus has previously been shown to exist in our Galaxy (Sawada et al. 2001). The wiggles in Fig. 14 can be associated with features in the $R_{21}$ map. The nucleus appears to be surrounded by a ring of excited gas, which is reflected as a bump in the radial distribution. The bar ends and inner arms are reflected as a flat region at $\approx 120-150^{\prime \prime}$, while the low $R_{21}$-values in the outer arms suppresses the average $R_{21}$-ratio in the range $200-300^{\prime \prime}$.

\section{Conclusions}

We have mapped, in over-sampling mode, the $\operatorname{CO}(J=1-0)$ and $\mathrm{CO}(J=2-1)$ brightness distributions over the entire optical disk of M 83. Significant corrections for an error beam have been applied to the $\mathrm{CO}(J=2-1)$ data. A MEM-method has been used to increase the angular resolution of the data.

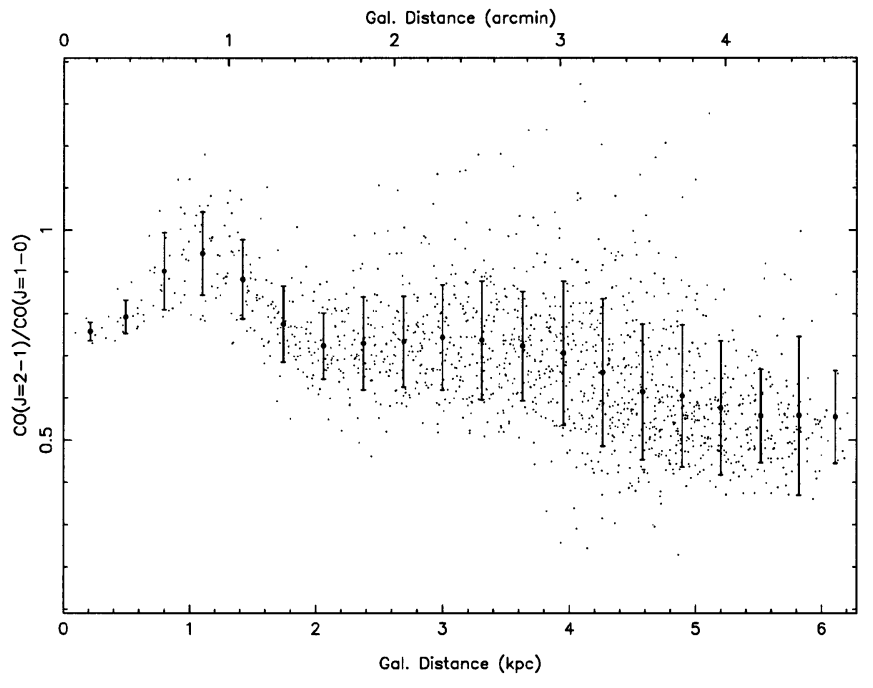

Fig. 14. The $\mathrm{CO}(J=2-1) /(J=1-0)$ line intensity ratio for individual positions (dots) and the azimuthally averaged ratio (with error bars) in annular zones in the plane of the galaxy.

We find that:

$i$ ) the $\mathrm{CO}$ emission is strongly peaked toward the nucleus, which splits up into two components in the $\mathrm{CO}(J=2-1)$ data. Also the bar ends are prominent. The $\mathrm{CO}$ emission follows the leading edges of the bar.

ii) Molecular gas spiral arms are clearly identified, and they trace, in most cases, the dust lanes. There are frequent "bridges" between spiral arms, and in some areas the arms are clearly disturbed.

iii) An average arm-interarm brightness contrast of about 2.5 is found for the $\operatorname{CO}(J=1-0)$ line.

iv) The $\mathrm{CO}(J=2-1) /(J=1-0)$ line ratio, which is about 0.77 on average, differ between the arm and interarm regions. It is significantly higher in the latter.

v) Regularly spaced molecular gas concentrations of mass $\approx 10^{7} M_{\odot}$ lie along the arms.

vi) The estimated total molecular gas mass is $3.9 \times 10^{9} M_{\odot}$, and within $7.3 \mathrm{kpc}$ the $\mathrm{H}_{2}$ mass dominates over that of $\mathrm{HI}$ by a factor of more than two. The estimated gas/stellar mass ratio is $\approx 0.1$ in the optical disk.

vii) The $\mathrm{CO}$ and $\mathrm{HI}$ emissions are very well correlated in the optical disk, and frequently concentrations of the two gas components coincide.

viii) The $\mathrm{CO}$ radial brightness distribution in the disk follows that of other starformation tracers as $\mathrm{H} \alpha$ emission, and continuum light in the $B$ and $K$ filters. The estimated scale length, of a fitted exponential, is about 120", corresponding to $2.6 \mathrm{kpc}$ at the adopted distance.

Acknowledgements. We are very grateful toward the SEST staff for their support during observations and Swedish Natural Science Research Council for travel expenses support. We also wish to thank Remo Tilanus and Ron Allen for letting us use their HI data and Sören Larsen for the use of the optical images and the referee for valuable input. 

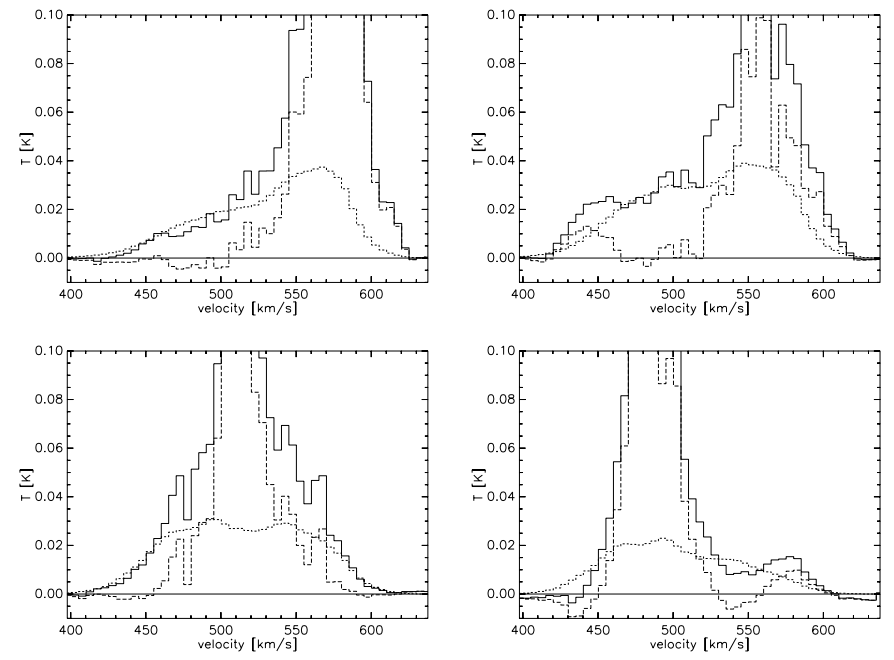

Fig. A.1. These spectra show the original spectra (solid lines), $200^{\prime \prime}$ convolved spectra (short-dashed lines) and subtracted spectra (long-dashed lines) in four typical positions. The coordinates with respect to the map center is, from upper left to lower right, $(-42,-70)$, $(-42,0),(-42,70)$ and $(-42,140)$. The $200^{\prime \prime}$ convolved spectra are scaled with the derived error beam efficiency.

\section{Appendix A: Error beam}

The beam pattern of a radio telescope is affected by its surface accuracy. This means that the power received in the error beam may very well be comparable to the power received in the main beam. For sources that are compact relative to the extent of the beam, it is relatively easy to compensate for this underestimate of the true flux: The measured antenna temperature $\left(T_{\mathrm{A}}^{*}\right)$ is simply divided by the main beam efficiency, $\eta_{\mathrm{mb}}$, to attain the main beam brightness temperature $\left(T_{\mathrm{mb}}\right)$. The main beam efficiency is usually well known and can be found in the user manual of the different telescopes. For extended sources, the situation is more complex, since the antenna may receive power not only from the main beam. If the size of the error beam is comparable to the extent of the source, a simple multiplication of $\left(\eta_{\mathrm{mb}}\right)^{-1}$ will overestimate the flux at the position where the measurement is done. If the antenna pattern is well known, it is possible to calculate how the beam couples to the source and thus compensate for this effect. Unfortunately, the antenna pattern for SEST has never been measured properly, so this was not an option for us. Instead we devised a procedure to remove the impact of the error beam, using our own data set to estimate the strength and size of the error beam pattern.

Nearly all $\operatorname{CO}(J=2-1)$ spectra did show two components: one narrow line, that agrees with the width and center velocity of the $\operatorname{CO}(J=1-0)$ spectra at the same position and another, broader, component that is picked up by the error beam. By studying these broad wings in a number of spectra in the deconvolved $\mathrm{CO}(J=2-1)$ data set and by comparing with spectra in data cubes convolved with different FWHPs, we estimated that the size of the error beam at $230 \mathrm{GHz}$ is of the order of $200^{\prime \prime}$. This is in good agreement with the error beam size of $3^{\prime}$, which was assumed by Johansson et al. (1998).

In order to try to estimate the impact of the error beam we produced a data set where we convolved the MEM-deconvolved data set with a $200^{\prime \prime}$ Gaussian profile. In each position an automated process, using the sliding window technique, calculated the velocity-integrated intensity in the region of the extended wings (i.e. outside the velocity range where we have seen emission in the $\operatorname{CO}(J=1-0)$ data) in both the deconvolved cube and in the $200^{\prime \prime}$ convolved cube. The ratio between these intensities corresponds to the fraction of the available emission which is picked up by the error beam. We will refer to this as the error beam efficiency. In principle, the error beam efficiency must lie in the interval $0.1-0.5$, since the main beam efficiency is 0.5 and the moon beam efficiency is 0.9 . Some values of our error beam efficiency estimates are higher than 0.5 . This happens in regions where the emission in the main beam falls outside the expected velocity range (e.g., in regions with exceptionally strong streaming motions or wide emission profiles), leading to an over-estimation of the flux in the wings. An attempt to make the window wider had a negative impact on the results since we were left with fewer channels to calculate the velocity-integrated emission in the wings. Neglecting values above 0.5 , we found that the error beam efficiency was on average $0.27 \pm 0.11$.

Figure A.1 shows the original spectrum, the $200^{\prime \prime}$ convolved spectrum (scaled with the error beam efficiency), and the resulting (cleaned) spectrum after subtraction of the two previous spectra, in four typical positions. The convolved spectra reproduces the shape of the wings very well, and the shape of the cleaned spectra resembles the corresponding $\mathrm{CO}(J=1-0)$ spectra (not shown).

We have corrected the convolved and the MEM-deconvolved data sets for this error by subtracting the error beam contribution for each individual spectrum. Based on the previous result, we took a conservative approach and fixed the error beam contribution to 0.27 . In principle, we could have let this ratio vary from spectrum to spectrum by fitting the $200^{\prime \prime}$-convolved spectrum to the extended wings, but this would have introduced other, not easily controlled, errors.

\section{References}

Aalto, S., Hüttemeister, S., Scoville, N. Z., \& Thaddeus, P. 1999, ApJ, 522,165

Adamson, A. J., Adams, D. J., \& Warwick, R. S. 1987, MNRAS, 224, 367

Arimoto, N., Sofue, Y., \& Tsujimoto, T. 1996, PASJ, 48, 275

Booth, R. S., Delgado, G., Hagstrom, M., et al. 1989, A\&A, 216, 315

Calzetti, D., Conselice, C. J., Gallagher, J. S., \& Kinney, A. L. 1999, AJ, 118, 797

Casoli, F., Sauty, S., Gerin, M., et al. 1998, A\&A, 331, 451

Combes, F. 1991, ARA\&A, 29, 195

Combes, F. 2000, in Euroconference, The Evolution of Galaxies, I- Observational Clues, ed. J. Vilchez, G. Stasinska, \& E. Perez, 7318

Combes, F., Encrenaz, P. J., Lucas, R., \& Weliachew, L. 1978, A\&A, 67, L13

Comerón, F. 2001, A\&A, 365, 417

Comte, G. 1981, A\&AS, 44, 441

Crosthwaite, L. P., Turner, J. L., Buchholz, L., Ho, P. T. P., \& Martin, R. N. 2002, AJ, 123, 1892

Crosthwaite, L. P., Turner, J. L., Hurt, R. L., et al. 2001, AJ, 122, 797

de Vaucouleurs, G. 1979, AJ, 84, 1270 
de Vaucouleurs, G., de Vaucouleurs, A., \& Corwin, H. G. 1976, Second Reference Catalogue of Bright Galaxies (RC2) (University of Texas Press)

de Vaucouleurs, G., de Vaucouleurs, A., Corwin, H. G., et al. 1992, VizieR Online Data Catalog, 7137

de Vaucouleurs, G., Pence, W. D., \& Davoust, E. 1983, ApJS, 53, 17

Elmegreen, D. M., Chromey, F. R., \& Warren, A. R. 1998, AJ, 116, 2834

Fitt, A. J., Howarth, N. A., Alexander, P., \& Lasenby, A. N. 1992, MNRAS, 255, 146

Garcia-Burillo, S., Guelin, M., \& Cernicharo, J. 1993, A\&A, 274, 123

Gibson, B. K., Stetson, P. B., Freedman, W. L., et al. 2000, ApJ, 529, 723

Handa, T., Ishizuki, S., \& Kawabe, R. 1994, in IAU Colloq. 140, Astronomy with Millimeter and Submillimeter Wave Interferometry, ASP Conf. Ser., 59, 341

Handa, T., Nakai, N., Sofue, Y., Hayashi, M., \& Fujimoto, M. 1990, PASJ, 42, 1

Helfer, T. T., Thornley, M. D., Regan, M. W., et al. 2003, ApJS, 145, 259

Houghton, S., Whiteoak, J. B., Koribalski, B., et al. 1997, A\&A, 325, 923

Huchtmeier, W. K., \& Bohnenstengel, H. 1981, A\&A, 100, 72

Immler, S., Vogler, A., Ehle, M., \& Pietsch, W. 1999, A\&A, 352, 415

Israel, F. P., \& Baas, F. 2001, A\&A, 371, 433

Jensen, E. B., Talbot, R. J., \& Dufour, R. J. 1981, ApJ, 243, 716

Johansson, L. E. B., Greve, A., Booth, R. S., et al. 1998, A\&A, 331, 857

Kenney, J. D. P., \& Lord, S. D. 1991, ApJ, 381, 118

Kenney, J. D. P., Scoville, N. Z., \& Wilson, C. D. 1991, ApJ, 366, 432

Kennicutt, R. C. 1989, ApJ, 344, 685

Knapp, G. R. 1987, PASP, 99, 1134

Kobulnicky, H. A., \& Skillman, E. D. 1995, ApJ, 454, L121

Kuno, N., Nishiyama, K., Nakai, N., et al. 2000, PASJ, 52, 775

Larsen, S. S., \& Richtler, T. 1999, A\&A, 345, 59

Loinard, L., Dame, T. M., Heyer, M. H., Lequeux, J., \& Thaddeus, P. 1999, A\&A, 351, 1087

Lord, S. D. 1987, Ph.D. Thesis, Massachusetts Univ., Amherst

Lord, S. D., \& Kenney, J. D. P. 1991, ApJ, 381, 130
Nakai, N., Kuno, N., Handa, T., \& Sofue, Y. 1994, PASJ, 46, 527

Neininger, N., Beck, R., Sukumar, S., \& Allen, R. J. 1993, A\&A, 274, 687

Nieten, C., Neininger, N., Guélin, M., et al. 2000, in The interstellar medium in M 31 and M 33, Proc. 232, WE-Heraeus Seminar, 21

Nishiyama, K., Nakai, N., \& Kuno, N. 2001, PASJ, 53, 757

Ondrechen, M. P. 1985, AJ, 90, 1474

Papadopoulos, P. P., \& Allen, M. L. 2000, ApJ, 537, 631

Parodi, B. R., Saha, A., Sandage, A., \& Tammann, G. A. 2000, ApJ, 540, 634

Petitpas, G. R., \& Wilson, C. D. 1998, ApJ, 503, 219

Rand, R. J., Lord, S. D., \& Higdon, J. L. 1999, ApJ, 513, 720

Regan, M. W., Thornley, M. D., Helfer, T. T., et al. 2001, ApJ, 561, 218

Rogstad, D. H., Lockart, I. A., \& Wright, M. C. H. 1974, ApJ, 193, 309

Rydbeck, G. 2000, in Imaging at Radio through Submillimeter Wavelengths, ASP Conf. Ser., 217, 224

Saha, A., Sandage, A., Labhardt, L., et al. 1995, ApJ, 438, 8

Sandage, A., \& Tammann, G. A. 1974, ApJ, 194, 559

Sawada, T., Hasegawa, T., Handa, T., et al. 2001, ApJS, 136, 189

Sofue, Y., \& Wakamatsu, K. 1994, AJ, 107, 1018

Soria, R., \& Wu, K. 2002, A\&A, 384, 99

Strong, A. W., Bloemen, J. B. G. M., Dame, T. M., et al. 1988, A\&A, 207,1

Tacconi, L. J., \& Young, J. S. 1989, ApJS, 71, 455

Talbot, R. J., Jensen, E. B., \& Dufour, R. J. 1979, ApJ, 229, 91

Thim, F., Tammann, G. A., Saha, A., et al. 2003, ApJ, 590, 256

Tilanus, R. P. J., \& Allen, R. J. 1993, A\&A, 274, 707

Wall, W. F. 1991, Ph.D. Thesis, Texas Univ., Austin

Wiklind, T., Rydbeck, G., Hjalmarson, A., \& Bergman, P. 1990, A\&A, 232, L11

Wilson, C. D. 1995, ApJ, 448, L97

Young, J. S. 1999, ApJ, 514, L87

Young, J. S., \& Rownd, B. K. 2001, in Galaxy Disks and Disk Galaxies, ASP Conf. Ser., 230, 391

Young, J. S., \& Scoville, N. Z. 1991, ARA\&A, 29, 581

Young, J. S., Xie, S., Tacconi, L., et al. 1995, ApJS, 98, 219 